\title{
SUFFICIENT CONDITIONS FOR A WEAK RELATIVE MINIMUM IN THE PROBLEM OF BOLZA
}

BY

\author{
E. J. McSHANE
}

1. Introduction. The past decade has brought forth great advances in the theory of the Bolza problem in the calculus of variations and in the theory of the problems (Lagrange, Mayer, and so on) subsumed under it. Ten years ago the necessary conditions of Weierstrass, Clebsch and Jacobi (or Mayer) were established only for minimizing curves normal on every subarc, while the sufficiency theorems needed even more drastic normality assumptions. Now the sufficiency theorems are established under the assumption that Lagrange multipliers $\lambda_{0} \geqq 0, \lambda_{1}(x), \cdots, \lambda_{m}(x)$ exist with which the curve $E_{12}$ satisfies the Euler equation, the transversality condition, and the strengthened Weierstrass, Clebsch and Jacobi conditions. The Euler equation, transversality condition, Weierstrass condition and Clebsch condition are proved necessary with no normality assumptions. Yet normality requirements have not been entirely dispensed with. I have shown $\left({ }^{1}\right)$ that for minimizing curves with order of abnormality 0 or 1 there are multipliers with which all the standard necessary conditions are satisfied. But an example shows that minimizing curves exist, having order of abnormality 2, which do not satisfy all the standard necessary conditions with any multipliers. Thus if the gap is to be closed and the necessary and sufficient conditions brought together for abnormal problems, our only hope is to strengthen the very strong sufficiency theorems of Hestenes, Morse, and Reid.

In a paper to be published in the American Mathematical Monthly, I have considered the problem of minimizing a function $f^{0}(x) \equiv f^{0}\left(x^{1}, \cdots, x^{n}\right)$ subject to conditions

$$
f^{\beta}(x)=0 \quad(\beta=1, \cdots, m) .
$$

Subject to fairly obvious conditions of definition and differentiability, I have shown that the following condition is necessary in order that $f^{0}$ have a minimum subject to (1.1) at a point $x_{0}$ satisfying (1.1).

(N) To each set $\left(u^{1}, \cdots, u^{n}\right)$ satisfying the conditions $\left({ }^{2}\right)$

$$
f_{x^{i}}^{\beta}\left(x_{0}\right) u^{i}=0 \quad(\beta=1, \cdots, m)
$$

Presented to the Society, May 2, 1941; received by the editors April 4, 1941, and, in revised form, October 11, 1941.

(1) On the second variation in certain abnormal problems of the calculus of variations, American Journal of Mathematics, vol. 63 (1941), pp. 516-530.

(2) We use the summation convention, summing on all repeated indices. 
there correspond multipliers $l_{0} \geqq 0, l_{1}, \cdots, l_{m}$ not all zero such that

$$
l_{\alpha} f_{x i}^{\alpha}\left(x_{0}\right)=0
$$

and

$$
l^{\alpha} f_{x^{i} x^{j}}\left(x_{0}\right) u^{i} u^{j} \geqq 0 .
$$

Correspondingly, we show that the following condition is sufficient for $f^{0}(x)$ to have a proper minimum subject to (1.1) at a point $x_{0}$ satisfying (1.1).

(S) To each set of numbers $\left(u^{1}, \cdots, u^{n}\right)$ not all zero satisfying (1.2) there corresponds a set of multipliers $l_{0} \geqq 0, l_{1}, \cdots, l_{m}$ such that (1.3) holds and the left member of (1.4) is positive.

Here we have no normality assumptions whatever, and still the gap between conditions $(\mathrm{N})$ and $(\mathrm{S})$ is no greater than that between the necessary condition and the sufficient conditions for a minimum of a function $f(x)$ of a single real variable, without side conditions. The distinctive feature of conditions $(\mathrm{N})$ and $(\mathrm{S})$ is the dependence of the multipliers $l_{0}, \cdots, l_{m}$ on the solutions $u^{1}, \cdots, u^{n}$ of equations (1.2).

Let us now consider the Bolza problem of minimizing a functional

$$
g\left(x_{1}, y\left(x_{1}\right), x_{2}, y\left(x_{2}\right)\right)+\int_{x_{1}}^{x_{2}} f\left(x, y, y^{\prime}\right) d x
$$

in the class of functions

$$
y^{i}=y^{i}(x) \quad\left(i=1, \cdots, n ; x_{1} \leqq x \leqq x_{2}\right)
$$

satisfying certain differential equations

$$
\phi^{\alpha}\left(x, y, y^{\prime}\right)=0 \quad \cdots \quad\left(\alpha=1, \cdots, m<n ; x_{1} \leqq x \leqq x_{2}\right)
$$

and certain end conditions

$$
\psi^{\mu}\left(x_{1}, y\left(x_{1}\right), x_{2}, y\left(x_{2}\right)\right)=0 \quad(\mu=1, \cdots, p \leqq 2 n+2) .
$$

Under the usual hypotheses on the functions, we are led by the theorems of the preceding paragraph to the following conjectures.

Conjecture (N). If a curve

$$
E_{12}: \quad y^{i}=y^{i}(x), \quad x_{1} \leqq x \leqq x_{2},
$$

minimizes the functional (1.5) in the class of curves satisfying (1.7) and (1.8) then for each set of functions $\eta^{i}(x)$ and numbers $\xi_{1}, \xi_{2}$ which satisfy the equations of variation of (1.7) and (1.8) there are multipliers $\lambda^{0} \geqq 0, \lambda^{\alpha}(x)$ not all zero such that for the function

$$
F\left(x, y, y^{\prime}, \lambda\right) \equiv \lambda^{0} f\left(x, y, y^{\prime}\right)+\lambda^{\alpha}(x) \phi^{\alpha}\left(x, y, y^{\prime}\right)
$$


the Euler equations, transversality condition, Weierstrass condition and Clebsch condition are satisfied, and the second variation formed in the usual way from $F$ and $\eta$ and the end functions is non-negative.

CONJECTURE (S). In order that a smooth curve (1.9) shall give a strong proper relative minimum to the functional (1.5) in the class of curves satisfying (1.7) and (1.8), it is sufficient that the following condition be satisfied. To each nonidentically zero set $\left[\eta^{i}(x), \xi_{1}, \xi_{2}\right]$ satisfying the equations of variation of (1.7) and (1.8) there shall correspond multipliers $\lambda^{0} \geqq 0, \lambda^{\alpha}(x)$ with which the Euler equation, transversality condition and strengthened Weierstrass and Clebsch conditions hold, and the second variation is positive.

These conjectures are now being investigated, the first by Miss Mary Jane Cox and the second by Mr. Franklin G. Myers $\left({ }^{3}\right)$. The purpose of the present paper is to establish the analogue of Conjecture (S) for weak relative minima. The proof is made by expansion methods, not as a matter of choice but rather as a matter of necessity. The field theory hardly seems applicable. We cannot even find a conjugate set of accessory extremals; worse, we cannot even set up an accessory problem, because of the dependence of the multipliers on the $\eta^{i}(x)$.

We shall obtain a sufficiency theorem for the parametric form of the problem, and from this we shall deduce a theorem for the non-parametric problem.

2. Statement of the problem. We shall study the Bolza problem in parametric form. On an open set $R_{1}$ of points $(y, r)=\left(y^{0}, \cdots, y^{n}, r^{0}, \cdots, r^{n}\right)$ in $(2 n+2)$-dimensional space we are given functions

$$
f\left(y, y^{\prime}\right), \quad \phi^{\beta}\left(y, y^{\prime}\right) \quad(\beta=1, \cdots, m<n)
$$

of class $C^{2}$. We assume that if $(y, r)$ is in $R_{1}$ so is $(y, k r)$ for all $k>0$, and that $f$ and the $\phi^{\beta}$ are positively homogeneous of degree 1 in $r$. Also, we are given functions

$$
\theta(\alpha), \quad T^{i s}(\alpha) \quad(i=0,1, \cdots, n ; s=1,2)
$$

defined and of class $C^{2}$ on an open set $R_{2}$ in an $r$-dimensional space of points $\left(\alpha^{1}, \cdots, \alpha^{r}\right)$.

If $C$ is a rectifiable curve, having a representation

$$
C: \quad y^{i}=y^{i}(t) \quad\left(t_{1} \leqq t \leqq t_{2} ; i=0,1, \cdots, n\right)
$$

with absolutely continuous functions $y^{i}(t)$, and $\alpha$ is a point in $R_{\mathbf{2}}$, we say that the set $(C, \alpha)$ is admissible, or that $C$ is admissible with parameters $\alpha$, if for almost all $t$ the point $(y, \dot{y})$ lies in $R_{1}$ and satisfies the equations

(3) Added in proof, June 1942: Miss Cox has established Conjecture N; Dr. Myers has shown that the hypotheses of Conjecture $\mathrm{S}$ yield a semi-strong minimum, and for certain integrands guarantee a strong minimum. 


$$
\phi^{\beta}(y(t), \dot{y}(t))=0 \quad(\beta=1, \cdots, m),
$$

and the end conditions

$$
y^{i}\left(t_{s}\right)=T^{i s}(\alpha) \quad(i=0,1, \cdots, n ; s=1,2)
$$

are satisfied.

The problem of Bolza is the problem of minimizing the functional

$$
J(C, \alpha)=\theta(\alpha)+\int_{t_{1}}^{t_{2}} f\left(y(t), y^{\prime}(t)\right) d t
$$

on the class of all admissible sets $(C, \alpha)$.

Following Carathéodory, we shall denote by $\dot{y}(t)$ the vector $\left(y^{0 \prime}(t), \cdots\right.$, $\left.y^{n \prime}(t)\right)$ if this vector is defined and finite, and the vector $(0, \cdots, 0)$ otherwise. We shall denote the length of a vector by enclosing the vector between vertical bars; thus

$$
\begin{aligned}
\left|y_{1}-y_{2}\right| & =\left\{\sum_{i=0}^{n}\left(y_{1}^{i}-y_{2}^{i}\right)^{2}\right\}^{1 / 2}, \\
|\alpha| & =\left\{\sum_{h=1}^{+}\left(\alpha^{h}\right)^{2}\right\}^{1 / 2} .
\end{aligned}
$$

The concept of weak relative minimum has been given several equivalent formulations. For simplicity of notation, let us suppose that $C_{0}$ is a curve of class $C^{1}$, represented by equations

$$
y^{i}=y_{0}^{i}(t) \quad\left(t_{1} \leqq t \leqq t_{2}\right)
$$

in which the functions $y^{i}(t)$ are of class $C^{1}$ and $\left|y^{\prime}\right| \neq 0$. A curve $C$ is in the first order $\epsilon$-neighborhood of $C_{0}$ if it has a Lipschitzian representation (2.1) such that

$$
\left|y(t)-y_{0}(t)\right|<\epsilon \quad\left(t_{1} \leqq t \leqq t_{2}\right)
$$

and

$$
\underset{t_{1} \leqq t_{\leqq} t_{2}}{\operatorname{l.u.b.}}\left|\left(y(t)-y_{0}(t)\right) \cdot\right| /\left|y_{0}^{\prime}(t)\right|<\epsilon .
$$

This neighborhood is easily seen to be independent of the particular representation of $C_{0}$.

We say that the functional $J(C, \alpha)$ has a weak relative minimum at the admissible set $\left(C_{0}, \alpha_{0}\right)$ if there is a positive number $\epsilon$ such that

$$
J(C, \alpha) \geqq J\left(C_{0}, \alpha_{0}\right)
$$

for all admissible sets $(C, \alpha)$ having $C$ in the first order $\epsilon$-neighborhood of $C_{0}$ 
and $\left|\alpha-\alpha_{0}\right|<\epsilon$. The minimum is proper if equality is excluded from (2.7) except when $(C, \alpha)=\left(C_{0}, \alpha_{0}\right)$. In this paper we shall set forth conditions which ensure that a set $\left(C_{0}, \alpha_{0}\right)$ gives a proper weak relative minimum to $J(C, \alpha)$.

3. Statement of the theorem. We use a slight modification of the summation convention. The repetition of an index in a term connotes the summation of the values of that term over all values of the repeated index, except that the indices $q$ and $s$ are exempted; we never sum over values of $q$ or of $s$. As usual, for each set of numbers $\lambda^{0}, \cdots, \lambda^{m}$ we define

$$
F(y, r, \lambda) \equiv \lambda^{0} f(y, r)+\lambda^{\beta} \phi^{\beta}(y, r) .
$$

Henceforth we suppose that $\left(C_{0}, \alpha_{0}\right)$ is an admissible set, the curve $C_{0}$ being represented by (2.5) with functions $y_{0}^{i}(t)$ of class $C^{1}$ which have $\left|y_{0}^{\prime}\right|>0$. The curve $C_{0}$ satisfies the Euler equations with multipliers $\lambda^{0}, \lambda^{1}(t), \cdots, \lambda^{m}(t)$ if

$$
\begin{array}{r}
\frac{d}{d t} F_{r^{i}}\left(y_{0}(t), y_{0}^{\prime}(t), \lambda\right)=F_{y^{i}}\left(y_{0}(t), y_{0}^{\prime}(t), \lambda\right) \\
\left(i=0,1, \cdots, n ; t_{1} \leqq t \leqq t_{2}\right) .
\end{array}
$$

The set $\left(C_{0}, \alpha_{0}\right)$ satisfies the transversality condition with multipliers $\lambda^{0}, \lambda^{1}(t), \cdots, \lambda^{m}(t)$ if

$$
\begin{aligned}
& \lambda^{0} \theta_{h}(\alpha)+F_{r i}\left(y_{0}\left(t_{2}\right), y_{0}^{\prime}\left(t_{2}\right), \lambda\left(t_{2}\right)\right) T_{h}^{i 2}\left(\alpha_{0}\right) \\
&-F_{r i}\left(y_{0}\left(t_{1}\right), y_{0}^{\prime}\left(t_{1}\right), \lambda\left(t_{1}\right)\right) T_{h}^{i 1}\left(\alpha_{0}\right)=0 \\
&(h=1, \cdots, r),
\end{aligned}
$$

the subscript $h$ denoting partial differentiation with respect to $\alpha^{h}$.

For the curve $C_{0}$ it is well known that the quadratic form

$$
F_{r i r} j\left(y_{0}(t), y_{0}^{\prime}(t), \lambda\right) v^{i} v^{j}
$$

vanishes whenever the vector $v$ is linearly dependent on $y_{0}^{\prime}(t)$; that is, whenever there is a number $k$ such that

$$
v^{i}=k y_{0}^{i \prime}(t) \quad(i=0,1, \cdots, n) .
$$

The curve $C_{0}$ is said to satisfy the strengthened Clebsch condition if for each $t$ in the interval (3.4) and every vector $v$ linearly independent of $y^{i \prime}(t)$ and satisfying the equations

$$
\phi_{r^{i}}^{\beta}\left(y_{0}(t), y_{0}^{\prime}(t)\right) v^{i}=0 \quad(\beta=1, \cdots, m)
$$

the quadratic form (3.4) is positive.

Our definition of admissible variations is somewhat more inclusive than the usual definition. We define an admissible variation set to be a set $[\eta(t), u]=\left[\eta^{0}(t), \cdots, \eta^{n}(t), u^{1}, \cdots, u^{h}\right]$ in which the functions $\eta^{i}(t)$ are ab- 
solutely continuous, have derivatives integrable together with their squares, and satisfy the equations of variations of (2.2), that is, the equations

$$
\begin{array}{r}
\Phi^{\beta}(t, \eta, \dot{\eta}) \equiv \phi_{y^{i}}^{\beta}\left(y_{0}(t), y_{0}^{\prime}(t)\right) \eta^{i}(t)+\phi_{r^{i}}^{\beta}\left(y_{0}(t), y_{0}^{\prime}(t)\right) \dot{\eta}^{i}(t)=0 \\
\left(\beta=1, \cdots, m ; t_{1} \leqq t \leqq t_{2}\right),
\end{array}
$$

for amost all values of $t$ in the interval $\left[t_{1}, t_{2}\right]$, and the numbers $u^{h}$ satisfy the equations of variation of (2.3), which are

$$
\eta^{i}\left(t_{s}\right)-T_{h}^{i s}\left(\alpha_{0}\right) u^{h}=0 \quad(s=1,2 ; i=0,1, \cdots, n) .
$$

If $[\eta, u]$ is an admissible variation set, and $\lambda^{0}, \cdots, \lambda^{m}(t)$ are multipliers, we define the second variation due to $[\eta, u]$ by the equation

$$
J_{2}[\eta, u, \lambda] \equiv b_{h k} u^{h} u^{k}+\int_{t_{1}}^{t_{2}} 2 \omega(t, \eta, \dot{\eta}) d t
$$

where

and

$$
\begin{aligned}
b_{h k}= & \theta_{h k}\left(\alpha_{0}\right)+F_{r^{i}}\left(y_{0}\left(t_{2}\right), y_{0}^{\prime}\left(t_{2}\right), \lambda\left(t_{2}\right)\right) T_{h k}^{i 2}\left(\alpha_{0}\right) \\
& -F_{r^{i}}\left(y_{0}\left(t_{1}\right), y_{0}^{\prime}\left(t_{1}\right), \lambda\left(t_{1}\right)\right) T_{h k}^{i 1}\left(\alpha_{0}\right)
\end{aligned}
$$

$$
2 \omega(t, \eta, \rho)=F_{y} y_{y} j \eta^{i} \eta^{j}+2 F_{y} i_{r} j \eta^{i} \rho^{j}+F_{r} i_{r} j \rho^{i} \rho^{j},
$$

the arguments of the functions in the right member being $\left(y_{0}(t), y_{0}^{\prime}(t), \lambda(t)\right)$.

An admissible variation set $[\eta, u]$ will be called essentially null if

$$
u^{h}=0 \quad(h=1, \cdots, r)
$$

and there is a function $\rho(t)$ such that

$$
\eta^{i}(t)=\rho(t) y_{0}^{i \prime}(t) \quad\left(t_{1} \leqq t \leqq t_{2} ; i=0,1, \cdots, n\right) .
$$

For such sets the second variation is known to have the value 0 .

We can now state our principal theorem.

THEOREM I. Let the following hypotheses be satisfied.

(1) The set $\left[C_{0}, \alpha_{0}\right]$ is admissible and the curve $C_{0}$ is a simple arc of class( $\left.{ }^{4}\right)$ $C^{2}$, represented by equation (2.5) with functions $y_{0}^{i}(t)$ of class $C^{2}$.

(2) For all $t$ in the interval $\left[t_{1}, t_{2}\right]$ the matrix

has rank $m$.

$$
\left\|\phi_{r i}^{\beta}\left(y_{0}(t), y_{0}^{\prime}(t)\right)\right\|
$$

(3) To each admissible variation set $[\eta, u]$ which is not essentially null there corresponds a set of continuous multipliers $\lambda^{0} \geqq 0, \lambda^{1}(t), \cdots, \lambda^{m}(t)$ with which

(4) If we assume $C$ to be of class $C^{1}$, in the presence of hypothesis (3) we can show by the Hilbert differentiability theorem that it must be of class $C^{2}$. 
the Euler equations, transversality condition and strengthened Clebsch condition are satisfied, and with which the inequality

$$
J_{2}(\eta, u, \lambda)>0
$$

holds.

Then $\left(C_{0}, \alpha_{0}\right)$ gives $J(C, \alpha)$ a proper weak relative minimum on the class of admissible sets $(C, \alpha)$.

4. A change of parameter. There is no loss of generality in assuming that (2.3) is the representation of $C_{0}$ in terms of arc length, so that $t_{1}=0$ and $t_{2}$ is the length of $C_{0}$ and

$$
\left|y_{0}^{\prime}(t)\right|=1 \quad\left(t_{1} \leqq t \leqq t_{2}\right) .
$$

Our proof will be indirect; we assume the theorem false, and arrive (in \$9) at a contradiction.

If the theorem is false, there exists a sequence of admissible sets $\left[C_{q}, \alpha_{q}\right]$ $(q=1,2,3, \cdots)$ with the following properties. Each set $\left(C_{q}, \alpha_{q}\right)$ is distinct from $\left(C_{0}, \alpha_{0}\right)$. The curves $C_{q}$ have Lipschitzian representations

$$
C_{q}: y^{i}=y_{q}^{i}(t)
$$

such that

$$
\lim _{q \rightarrow \infty} y_{q}^{i}(t)=y_{0}^{i}(t)
$$

uniformly in $\left[t_{1}, t_{2}\right]$ and

$$
\lim _{q \rightarrow \infty}\left(y_{q}^{i}(t)-y_{0}^{i}(t)\right) \cdot=0
$$

uniformly in $\left[t_{1}, t_{2}\right]$. The $\alpha_{q}$ satisfy

$$
\lim _{q \rightarrow \infty} \alpha_{q}^{h}=\alpha_{0}^{h} \quad(h=1, \cdots, r) .
$$

And finally

$$
J\left(C_{q}, \alpha_{q}\right) \leqq J\left(C_{0}, \alpha_{0}\right) \quad(q=1,2, \cdots) .
$$

For convenience in our proofs it is highly desirable to choose a particular type of representation of the curves $C_{q}$. Specifically, we shall prove the following statement.

(4.7) There is no loss of generality in assuming that the representations (4.2) satisfy the equations

$$
\left[y_{q}^{i}(t)-y_{0}^{i}(t)\right] y_{0}^{i \prime}(t)=A_{q} t+B_{q}
$$

where $A_{q}$ and $B_{q}$ are constants. 
The range of definition of the functions $y_{0}^{t}(t)$ may easily be extended to an interval $t_{1}-\epsilon<t<t_{2}+\epsilon$ in such a way that they remain of class $C^{2}$ and the curve $y^{i}=y_{0}^{i}(t)$ remains a simple arc. Consider the equations

$$
\left[y^{i}-y_{0}^{i}(t)\right] y_{0}^{i \prime}(t)-A t-B=0 .
$$

The equations have the initial solutions

$$
y^{i}=y_{0}^{i}(t) ; \quad t_{1} \leqq t \leqq t_{2} ; \quad A=B=0 .
$$

On a neighborhood of the set (4.10) the left member of (4.9) is of class $C^{1}$; on the set (4.10) the partial derivative of the left member with respect to $t$ has the value 1 . Hence by a known theorem on implicit functions the equation (4.9) has a solution

$$
t=t(y, A, B)
$$

defined and of class $C^{1}$ for all $(y, A, B)$ in a set

$$
|A|<\delta, \quad|B|<\delta, \quad\left|y-y_{0}(t)\right|<\delta \quad\left(t_{1} \leqq t \leqq t_{2} ; \delta>0\right)
$$

and assuming values in the interval $\left(t_{1}-\epsilon, t_{2}+\epsilon\right)$.

The constants $A_{q}, B_{q}$ of (4.8) are determined by giving $t$ the values $t_{1}, t_{2}$. By (4.1) and (4.3) the left member of (4.8) approaches zero uniformly, hence $A_{q}$ and $B_{q}$ both tend to zero as $q \rightarrow \infty$. We may therefore assume

$$
\left|A_{q}\right|<\delta, \quad\left|B_{q}\right|<\delta
$$

for all $q$. For all but a finite number of values of $q$, which we discard, each point $y_{q}(\tau)\left(t_{1} \leqq \tau \leqq t_{2}\right)$ lies within a distance $\delta$ of some point of $C$. Hence the functions

$$
t_{q}(\tau) \equiv t\left(y_{q}(\tau), A_{q}, B_{q}\right) \quad\left(t_{1} \leqq \tau \leqq t_{2}\right)
$$

are defined and satisfy the equation

$$
\left[y_{q}^{i}(\tau)-y_{0}^{i}\left(t_{q}(\tau)\right)\right] y_{0}^{i}\left(t_{q}(\tau)\right)=A_{q} t_{q}(\tau)+B_{q} .
$$

The functions $t_{q}(\tau)$ are clearly Lipschitzian. The constants $A_{q}$ and $B_{q}$ were so chosen that equation (4.8) is satisfied at $t_{1}$ and $t_{2}$, whence

$$
t_{q}\left(t_{1}\right)=t_{1}, \quad t_{q}\left(t_{2}\right)=t_{2}
$$

By (4.3), $y_{q}(\tau)$ tends to $y_{0}(\tau)$ uniformly in the interval $\left[t_{1}, t_{2}\right]$, so by the definition (4.14) we have

$$
\lim _{q \rightarrow \infty} t_{q}(\tau)=t\left(y_{0}(\tau), 0,0\right)=\tau
$$

uniformly for $t_{1} \leqq \tau \leqq t_{2}$. Since

$$
\left|y_{q}(\tau)-y_{0}\left(t_{q}(\tau)\right)\right| \leqq\left|y_{q}(\tau)-y_{0}(\tau)\right|+\left|y_{0}(\tau)-y_{0}\left(t_{q}(\tau)\right)\right|
$$


relations (4.3) and (4.17) imply

$$
\lim _{a \rightarrow \infty}\left|y_{q}(\tau)-y_{0}\left(t_{q}(\tau)\right)\right|=0
$$

uniformly on $t_{1} \leqq \tau \leqq t_{2}$.

Let $M_{q}$ be the set of values of $\tau$ in $t_{1} \leqq \tau \leqq t_{2}$ for which $y_{q}^{\prime}(\tau)$ is defined; this set constitutes almost all of the interval $t_{1} \leqq \tau \leqq t_{2}$. On this set the function $t_{q}(\tau)$ also has a derivative, as we see from (4.14). For all $\tau$ in $M_{q}$ the inequality

$$
\left|y_{q}^{\prime}(\tau)-y_{0}^{\prime}\left(t_{q}(\tau)\right)\right| \leqq\left|y_{q}^{\prime}(\tau)-y_{0}^{\prime}(\tau)\right|+\left|y_{0}^{\prime}(\tau)-y_{0}^{\prime}\left(t_{q}(\tau)\right)\right|
$$

holds. So if $\gamma$ is an arbitrary positive number, for all large $q$ the inequality

$$
\left|y_{q}^{\prime}(\tau)-y_{0}^{\prime}\left(t_{q}(\tau)\right)\right|<\gamma
$$

holds on $M_{q}$, as follows from (4.19), (4.4), (4.17) and the continuity of $y_{0}^{\prime}$.

By differentiating both members of (4.15) we find that on $M_{q}$ the equation

$$
\left[A_{q}+1-\left\{y_{q}^{i}(\tau)-y_{0}^{i}\left(t_{q}(\tau)\right)\right\} y_{0}^{i \prime \prime}\left(t_{q}(\tau)\right)\right] t_{q}^{\prime}(\tau)=y_{q}^{i \prime}(\tau) y_{0}^{{ }^{\prime \prime}}\left(t_{q}(\tau)\right)
$$

holds. Let $\eta$ be an arbitrary positive number less than 1 . Since $A_{q}$ tends to zero and (4.18) holds, the quantity in square brackets in (4.21) lies between $(1+\eta / 2 n)^{-1 / 2}$ and $(1+\eta / 2 n)^{1 / 2}$ for all sufficiently large $q$. Since $\left|y_{0}^{\prime}\right|=1$, by (4.20) with proper choice of $\left.{ }^{5}\right) \gamma$ the right member of (4.21) lies between the same bounds for all large $q$. Hence for all sufficiently large $q$ we have

$$
\left(1+\frac{\eta}{2 n}\right)^{-1}<t_{q}^{\prime}(\tau)<1+\frac{\eta}{2 n}
$$

In particular, if we choose $\eta=1$ we find that for all but a finite number of values of $q$ (which we discard from further consideration) the value of $t_{q}^{\prime}(\tau)$ exceeds $2 / 3$ on $M_{q}$, which is almost all of $t_{1} \leqq \tau \leqq t_{2}$. Hence $t_{q}(\tau)$ has a Lipschitzian inverse; we denote it by $\tau_{q}(t)$. By (4.16) we see that

$$
\tau_{q}\left(t_{1}\right)=t_{1}, \quad \tau_{q}\left(t_{2}\right)=t_{2} .
$$

If $N_{q}$ is the image of $M_{q}$ under the mapping $t=t_{q}(\tau)$, then $N_{q}$ constitutes almost all of $t_{1} \leqq t \leqq t_{2}$. On it the derivative of $\tau_{q}(t)$ exists and is the reciprocal of the derivative of $t_{q}(\tau)$, so by (4.22)

$$
\left(1+\frac{\eta}{2 n}\right)^{-1}<\tau_{q}^{\prime}(t)<1+\frac{\eta}{2 n}
$$

for all $t$ in $N_{q}, q$ sufficiently large.

We now show that the equations

$$
y^{i}=y_{q}^{i}\left(\tau_{q}(t)\right)
$$

(5) The choice $\gamma=1-(1+\eta / 2 n)^{-1 / 2}$ will serve. 
form the desired representation of $C_{q}$. By (4.15) the equation (4.8) is satisfied. By (4.18), the new functions satisfy the convergence condition (4.3). Let $\eta$ be an arbitrary number between 0 and 1 . In $(4.20)$ we choose $\gamma=\eta / 4 n$. For all large $q$ inequalities (4.20) and (4.23) hold on $N_{q}$; and $t$ being arc length on $y_{0}$, we have

$$
\begin{aligned}
\left|\frac{d}{d t}\left[y_{q}\left(\tau_{q}(t)\right)-y_{0}(t)\right]\right| & =\left|y_{q}^{\prime}\left(\tau_{q}(t)\right) \tau_{q}^{\prime}(t)-y_{0}^{\prime}(t)\right| \\
& \leqq\left|y_{q}^{\prime}\left(\tau_{q}(t)\right)-y_{0}^{\prime}(t)\right| \tau_{q}^{\prime}(t)+\left|\tau_{q}^{\prime}(t)-1\right| \\
& <\gamma\left(1+\frac{\eta}{2 n}\right)+\frac{\eta}{2 n} \\
& <\frac{\eta}{n} .
\end{aligned}
$$

By integrating we see that each component

$$
y_{q}^{i}\left(\tau_{q}(t)\right)-y_{0}^{i}(t)
$$

satisfies a Lipschitz condition of constant $\eta / n$, so the derivative has absolute value at most $\eta / n$ where it is defined. Thus

$$
\left|\left\{y_{q}\left(\tau_{q}(t)\right)-y_{0}(t)\right\} \cdot\right| \leqq \eta
$$

whenever all the derivatives are defined. Elsewhere inequality (4.24) is trivial, the left member being zero. That is, the left member of (4.24) tends uniformly to zero as $q \rightarrow \infty$, completing the proof.

5. A convergence lemma. For each $q$ we define a non-negative number $k_{q}$ by the equation

$$
k_{q}^{2}=\left|\alpha_{q}-\alpha_{0}\right|^{2}+\left(\max \left|y_{q}(t)-y_{0}(t)\right|\right)^{2}+\int_{t_{1}}^{t_{2}}\left|\dot{y}_{q}(t)-y_{0}^{\prime}(t)\right|^{2} d t .
$$

These numbers are actually positive; otherwise $\left(C_{q}, \alpha_{q}\right)$ would be identical with $\left(C_{0}, \alpha_{0}\right)$, contrary to hypothesis. Next we define

$$
\begin{aligned}
u_{q}^{h}= & \left(\alpha_{q}^{h}-\alpha_{0}^{h}\right) / k_{q}(h=1, \cdots, r ; q=1,2, \cdots), \\
\eta_{q}^{i}(t)= & \left(y_{q}^{i}(t)-y_{0}^{i}(t)\right) / k_{q} \\
& \left(i=0,1, \cdots, n ; q=1,2, \cdots ; t_{1} \leqq t \leqq t_{2}\right) .
\end{aligned}
$$

From the preceding equations we have at once for each $q$ the equation

$$
\left|u_{q}\right|^{2}+\left(\max \left|\eta_{q}(t)\right|\right)^{2}+\int_{t_{1}}^{t_{2}}\left|\dot{\eta}_{q}(t)\right|^{2} d t=1,
$$

so that each summand on the left is at most 1 . By the Bolzano-Weierstrass 
theorem we can select a subsequence of the $u_{q}$ which converges to a limit $u_{0}$. There is no loss of generality in supposing that $\left\{u_{q}\right\}$ is already such a sequence, so that

$$
\lim _{q \rightarrow \infty} u_{q}^{h}=u_{0}^{h} \quad \quad(h=1, \cdots, r) .
$$

Let $\left(\alpha_{1}, \beta_{1}\right), \cdots,\left(\alpha_{l}, \beta_{l}\right)$ be a set of nonoverlapping subintervals of $\left[t_{1}, t_{2}\right]$, and let $E$ be the set consisting of the sum of these intervals. Then by the inequality of Schwarz

$$
\begin{aligned}
\sum_{j=1}^{l}\left|\eta_{q}\left(\beta_{j}\right)-\eta_{q}\left(\alpha_{j}\right)\right| & =\sum_{1}^{l}\left|\int_{\alpha_{j}}^{\beta_{j}} \dot{\eta}_{q} d t\right| \\
& \leqq \int_{E}\left|\dot{\eta}_{q}\right| d t \leqq\left[\int_{E}\left|\dot{\eta}_{q}\right|^{2} d t\right]^{1 / 2} \cdot\left[\int_{E} 1 d t\right]^{1 / 2} \\
& \leqq 1 \cdot[m E]^{1 / 2}=\left[\sum_{1}^{l}\left(\beta_{i}-\alpha_{i}\right)\right]^{1 / 2}
\end{aligned}
$$

In particular, letting $l=1$ we see that the $\eta_{q}$ are equi-continuous. Since they have the uniform bound 1 by (5.4), we know by Ascoli's theorem that the sequence $\left\{\eta_{q}\right\}$ contains a subsequence converging uniformly to a limit function $\eta_{0}(t)$. We may suppose that $\left\{\eta_{q}\right\}$ is already such a subsequence, so that

$$
\lim _{Q \rightarrow \infty} \eta_{q}^{i}(t)=\eta_{0}^{i}(t) \quad(i=0,1, \cdots, n)
$$

uniformly on the interval $\left[t_{1}, t_{2}\right]$ :

If we let $q$ tend to $\infty$ in (5.6) we obtain

$$
\sum_{j=1}^{l}\left|\eta_{0}\left(\beta_{j}\right)-\eta_{0}\left(\alpha_{j}\right)\right| \leqq\left[\sum_{j=1}^{l}\left(\beta_{j}-\alpha_{j}\right)\right]^{1 / 2},
$$

so that the functions $\eta_{0}^{i}(t)$ are absolutely continuous. We wish now to show that their derivatives have integrable squares.

LEMMA 1(6). Under the hypotheses on the $\eta_{q}$, the squares of the derivatives of the $\eta_{0}^{i}(t)$ are summable, and

$$
\int_{t_{1}}^{t_{2}}\left|\dot{\eta}_{0}\right|^{2} d t \leqq \liminf _{Q \rightarrow \infty} \int_{t_{1}}^{t_{2}}\left|\dot{\eta}_{q}\right|^{2} d t \leqq 1 .
$$

(6) This is in fact a corollary of almost any theorem on semi-continuity of integrals in nonparametric form. Lemma 3 is also a consequence of known theorems. But it seems preferable to give the fairly simple proofs of these lemmas rather than refer the reader to some exposition containing complications not essential for our present needs. 
For each positive integer $k$ we subdivide the interval $\left[t_{1}, t_{2}\right]$ into $2^{k}$ equal subintervals by points

$$
t_{1}=\tau_{1}<\tau_{2}<\cdots<\tau_{2 k_{+1}}=t_{2},
$$

and define piecewise linear functions $p_{k}^{i}(t)$ which coincide with $\eta^{i}(t)$ at each $\tau_{l}\left(l=1, \cdots, 2^{k}+1\right)$ and are linear between. Since the derivatives of these functions are constant on each subinterval we find

$$
\int_{t_{1}}^{t_{2}}\left|\dot{p}_{k}\right|^{2} d t=\sum_{l=1}^{2 k}\left|\eta_{0}\left(\tau_{l+1}\right)-\eta_{0}\left(\tau_{l}\right)\right|^{2} /\left(\tau_{l+1}-\tau_{l}\right) .
$$

By Schwarz' inequality,

$$
\left|\eta_{q}\left(\tau_{l+1}\right)-\eta_{q}\left(\tau_{l}\right)\right|^{2} \leqq\left[\int_{\tau_{l}}^{\tau_{l+1}}\left|\dot{\eta}_{q}\right| d t\right]^{2} \leqq\left(\tau_{l+1}-\tau_{l}\right) \int_{\tau_{l}}^{\tau_{l+1}}\left|\dot{\eta}_{q}\right|^{2} d t
$$

Hence

$$
\liminf _{a \rightarrow \infty} \int_{\tau_{l}}^{\tau_{l+1}}\left|\dot{\eta}_{q}\right|^{2} d t \geqq\left|\eta_{0}\left(\tau_{l+1}\right)-\eta_{0}\left(\tau_{l}\right)\right|^{2} /\left(\tau_{l+1^{*}}-\tau_{l}\right) .
$$

This, with (5.10), yields

$$
\liminf _{a \rightarrow \infty} \int_{t_{1}}^{t_{2}}\left|\dot{\eta}_{q}\right|^{2} d t \geqq \int_{t_{1}}^{t_{2}}\left|\dot{p}_{k}\right|^{2} d t .
$$

The integrand on the right is non-negative, and except on the set of measure zero on which one or more of the functions $\eta_{0}, p_{1}, \ldots$ are non-differentiable its limit as $k \rightarrow \infty$ is $\left|\dot{\eta}_{0}(t)\right|^{2}$. By Fatou's lemma,

$$
\liminf _{a \rightarrow \infty} \int_{t_{1}}^{t_{2}}\left|\dot{\eta}_{q}\right|^{2} d t \geqq \liminf _{k \rightarrow \infty} \int_{t_{1}}^{t_{2}}\left|\dot{p}_{k}\right|^{2} d t \geqq \int_{t_{1}}^{t_{2}}\left|\dot{\eta}_{0}\right|^{2} d t
$$

This establishes the lemma.

6. The equations of variation. In order to show that the $\eta_{0}(t)$ satisfy the equations of variation (3.6) it is convenient to prove a lemma.

LEMMA 2. If $g(t)$ is summable together with its square on the interval $\left[t_{1}, t_{2}\right]$, then

$$
\begin{aligned}
\lim _{q \rightarrow \infty} \int_{t_{1}}^{t_{2}} g(t)\left(\eta_{q}^{i}-\eta_{0}^{i}\right) d t=\lim _{q \rightarrow \infty} \int_{t_{1}}^{t_{2}} g(t)\left(\dot{\eta}_{q}^{i}-\dot{\eta_{0}}\right) d t=0 \\
(i=0,1, \cdots, n) .
\end{aligned}
$$

The vanishing of the first limit in (6.1) is easily established, for

$$
\left|\int_{t_{1}}^{t_{2}} g(t)\left(\eta_{q}^{i}-\eta_{0}^{i}\right) d t\right| \leqq \max \left|\eta_{q}^{i}-\eta_{0}^{i}\right| \cdot \int_{t_{1}}^{t_{2}}|g| d t
$$


and the right member tends to zero by (5.9). Consider then the other limit. Let $\epsilon$ be an arbitrary positive number. As is well known, it is possible to find a polynomial $p(t)$ such that

$$
\int_{t_{1}}^{t_{2}}(g(t)-p(t))^{2} d t<\epsilon^{2} / 16
$$

By (5.4) and (5.8), we have

$$
\left(\int_{t_{1}}^{t_{2}}\left(\dot{\eta}_{m}^{i}\right)^{2} d t\right)^{1 / 2} \leqq 1 \quad(m=0,1,2, \cdots) .
$$

So by Minkowski's inequality

$$
\left(\int_{t_{1}}^{t_{2}}\left(\dot{\eta}_{q}^{i}-\dot{\eta}_{0}^{i}\right)^{2} d t\right)^{1 / 2} \leqq 2 . \quad(q=1,2, \cdots) .
$$

From this and Schwarz' inequality we obtain

$$
\begin{aligned}
\mid \int_{t_{1}}^{t_{2}} g \cdot\left(\dot{\eta}_{q}^{i}-\dot{\eta}_{0}^{i}\right) d t & -\int_{t_{1}}^{t_{2}} p \cdot\left(\dot{\eta}_{q}^{i}-\dot{\eta}_{0}^{i}\right) d t \mid \\
& \leqq\left\{\int_{t_{1}}^{t_{2}}(g-p)^{2} d t\right\}^{1 / 2}\left\{\int_{t_{1}}^{t_{2}}\left(\dot{\eta}_{q}^{i}-\dot{\eta}_{0}^{i}\right)^{2} d t\right\}^{1 / 2}<\epsilon / 2 .
\end{aligned}
$$

By integration by parts,

$$
\int_{t_{1}}^{t_{2}} p(t)\left(\dot{\eta}_{q}^{i}-\dot{\eta}_{0}^{i}\right) d t=\left.p(t)\left(\dot{\eta}_{q}^{i}-\eta_{0}^{i}\right)\right|_{t_{2}} ^{t_{1}}-\int_{t_{1}}^{t_{2}} p^{\prime}(t)\left(\eta_{q}^{i}-\eta_{0}^{i}\right) d t
$$

The first term on the right tends to zero by (5.7), and the second tends to zero by the part of the lemma already proved. Therefore for all $q$ greater than a certain $q_{\mathrm{e}}$ we have

$$
\left|\int_{t_{1}}^{t_{2}} p(t)\left(\dot{\eta}_{q}^{i}-\dot{\eta}_{0}^{i}\right) d t\right|<\epsilon / 2
$$

Now (6.4) and (6.6) imply

$$
\left|\int_{t_{1}}^{t_{2}} g(t)\left(\dot{\eta}_{q}^{i}-\dot{\eta}_{0}^{i}\right) d t\right|<\epsilon
$$

if $q>q_{\epsilon}$, and our lemma is established.

The curves $C_{0}$ and $C_{q}$ are admissible; so the equations

$$
\phi^{\beta}\left(y_{q}(t), \dot{y}_{q}(t)\right)=\phi^{\beta}\left(y_{0}(t), y_{0}^{\prime}(t)\right)=0
$$

hold for almost all $t$ in the interval $\left[t_{1}, t_{2}\right]$. Hence, recalling (5.3), the equation

$$
A_{q}^{\beta, i}(t) \eta_{q}^{i}(t)+B_{q}^{\beta, i}(t) \dot{\eta}_{q}^{i}(t)=0
$$


holds for almost all $t$, the coefficients being defined by equations

$$
\begin{aligned}
& A_{q}^{\beta, i}(t)=\int_{0}^{1} \phi_{y^{i}}^{\beta}\left(y_{0}(t)+\tau\left[y_{q}(t)-y_{0}(t)\right], y_{0}^{\prime}(t)+\tau\left[\dot{y}_{q}(t)-y_{0}^{\prime}(t)\right]\right) d \tau, \\
& B_{q}^{\beta, i}(t)=\int_{0}^{1} \phi_{r^{i}}^{\beta}\left(y_{0}+\tau\left[y_{q}-y_{0}\right], y_{0}^{\prime}+\tau\left[\dot{y}_{q}-y_{0}^{\prime}\right]\right) d \tau .
\end{aligned}
$$

From (4.3) and (4.4) we deduce

$$
\begin{aligned}
& \lim _{q \rightarrow \infty} A_{q}^{\beta, i}(t)=\phi_{y^{i}}^{\beta}\left(y_{0}(t), y_{0}^{\prime}(t)\right), \\
& \lim _{q \rightarrow \infty} B_{q}^{\beta, i}(t)=\phi_{r^{i}}^{\beta}\left(y_{0}(t), y_{0}^{\prime}(t)\right)
\end{aligned}
$$

uniformly on $t_{1} \leqq t \leqq t_{2}$. For each such $t$ we have, by (6.8),

$$
\begin{aligned}
\int_{t_{1}}^{t}\left\{A_{q}^{\beta, i}(t) \eta_{q}^{i}(t)+\phi_{r^{i}}^{\beta}\left(y_{0}, y_{0}^{\prime}\right) \dot{\eta}_{q}^{i}(t)\right\} d t & \\
& =\int_{t_{1}}^{t_{2}}\left\{\dot{\phi}_{r^{i}}^{\beta}\left(y_{0}, y_{0}^{\prime}\right)-B_{q}^{\beta, i}(t)\right\}{\dot{\eta_{q}}}^{i}(t) d t .
\end{aligned}
$$

By the Schwarz inequality, with (5.4) and (6.11), the right member of (6.12) approaches 0 as $q \rightarrow \infty$. The limit of the left member is readily found with the help of (6.10) and Lemma 2 ; we obtain

$$
\int_{t_{1}}^{t}\left\{\phi_{y^{i}}^{\beta}\left(y_{0}(t), y_{0}^{\prime}(t)\right) \eta_{0}^{i}(t)+\phi_{r i \eta_{0}}^{\beta}\right\} d t=0 .
$$

(Here and henceforth we indicate the arguments only in the first function in a bracketed expression whenever the remaining terms have the same arguments.) By differentiating both members of (6.13) we find that $\eta_{0}(t)$ satisfies equations (3.6) for almost all $t$ in the interval $t_{1} \leqq t \leqq t_{2}$.

Since the sets $\left(C_{0}, \alpha_{0}\right)$ and $\left(C_{q}, \alpha_{q}\right)$ are admissible, the equations

$$
\begin{aligned}
& y_{0}^{i}\left(t_{s}\right)=T^{i s}\left(\alpha_{0}\right) \\
& y_{q}^{i}\left(t_{s}\right)=T^{i s}\left(\alpha_{q}\right)
\end{aligned} \quad(i=0,1, \cdots, n ; s=1,2)
$$

are satisfied. With (5.2) and (5.3), this implies

$$
k_{q} \eta_{q}^{i}\left(t_{s}\right)=T^{i s}\left(\alpha_{0}+k_{q} u_{q}\right)-T^{i s}\left(\alpha_{0}\right) .
$$

So by the theorem of mean value there is an $\bar{\alpha}_{q}$ on the line segment joining $\alpha_{0}$ and $\alpha_{q}$ such that

$$
\eta_{q}^{i}\left(t_{s}\right)=T_{h}^{i s}\left(\bar{\alpha}_{q}\right) u_{q}^{h}
$$


By passage to the limit we find

$$
\eta_{0}^{i}\left(t_{s}\right)=T_{h}^{i s}\left(\alpha_{0}\right) u_{0}^{h} \quad(i=0,1, \cdots, n ; s=1,2) .
$$

Thus it has been shown that $\left[\eta_{0}, u_{0}\right]$ is an admissible set, as defined in $\S 3$.

7. A semi-continuity proof. In the course of our proof we shall have need of a generalization of Lemma 1 . Let us suppose that $a_{i j}(t), b_{i j}(t), c_{i j}(t)$ $(i, j=0,1, \cdots, n)$ are functions defined and continuous on the interval $t_{1} \leqq t \leqq t_{2}$. We define

$$
I(\eta)=\int_{t_{1}}^{t_{2}}\left[a_{i j}(t) \eta^{i}(t) \eta^{i}(t)+2 b_{i j} \eta^{i} \dot{\eta}^{j}+c_{i j} \dot{\eta}^{i} \dot{\eta}^{i}\right] d t .
$$

Concerning such integrals we prove a sequence of three lemmas, the last of which is the one needed later.

LEMMA 3. If for each $t$ in the interval $\left[t_{1}, t_{2}\right]$ the quadratic form $c_{i} v^{2} v^{j}$ is positive definite, then

$$
\liminf _{q \rightarrow \infty} I\left(\eta_{q}\right) \geqq I\left(\eta_{0}\right) .
$$

It is evident that there is no loss of generality in assuming

$$
a_{i j}(t)=a_{j i}(t), \quad c_{i j}(t)=c_{j i}(t) \quad\left(t_{1} \leqq t \leqq t_{2} ; i, j=0,1, \cdots, n\right) .
$$

We readily compute

$$
\begin{aligned}
& a_{i} \eta_{q}^{i} \eta_{q}^{j}+2 b_{i j} \eta_{q}^{i} \dot{\eta}_{q}^{j}+c_{i} \dot{\eta}_{q}^{i} \dot{\eta}_{q}^{j}=a_{i} \eta_{0} \eta_{0}^{j}+2 b_{i j} \dot{\eta}_{0}^{i} \dot{\eta}_{0}^{j}+c_{i} \dot{\eta}_{0} \dot{\eta}_{0}^{j} \\
& +2\left(a_{i j} \eta_{0}^{j}+b_{i j} \dot{\eta}_{0}^{j}\right)\left(\eta_{q}^{i}-\eta_{0}^{i}\right) \\
& +2\left(b_{j i} \eta_{0}^{j}+c_{i} \dot{\eta}_{0}^{j}\right)\left(\dot{\eta}_{q}^{i}-\dot{\eta}_{0}^{i}\right) \\
& +a_{i j}\left(\eta_{q}^{i}-\eta_{0}^{i}\right)\left(\eta_{q}^{j}-\eta_{0}^{j}\right) \\
& +2 b_{i j}\left(\eta_{q}^{i}-\eta_{0}^{i}\right)\left(\dot{\eta}_{q}^{j}-\dot{\eta}_{0}^{j}\right) \\
& +c_{i j}\left(\dot{\eta}_{q}^{i}-\dot{\eta}_{0}^{i}\right)\left(\dot{\eta}_{q}^{j}-\dot{\eta}_{0}^{j}\right) \text {. }
\end{aligned}
$$

The last term is non-negative, hence

$$
\begin{aligned}
I\left(\eta_{q}\right) \geqq I\left(\eta_{0}\right) & +2 \int_{t_{1}}^{t_{2}}\left(a_{i j} \eta_{0}^{j}+b_{i j} \dot{\eta}_{0}^{j}\right)\left(\eta_{q}^{i}-\eta_{0}^{i}\right) d t \\
& +2 \int_{t_{1}}^{t_{2}}\left(b_{j i} \eta_{0}^{j}+c_{i j} \dot{\eta}_{0}^{j}\right)\left(\dot{\eta}_{q}^{i}-\dot{\eta}_{0}^{i}\right) d t \\
& +\int_{t_{1}}^{t_{2}} a_{i j}\left(\eta_{q}^{i}-\eta_{0}^{i}\right)\left(\eta_{q}^{j}-\eta_{0}^{j}\right) d t \\
& +2 \int_{t_{1}}^{t_{2}} b_{i j}\left(\eta_{q}^{i}-\eta_{0}^{i}\right)\left(\dot{\eta}_{q}^{j}-\dot{\eta}_{0}^{j}\right) d t .
\end{aligned}
$$


The second and third terms in the right member approach zero by Lemma 2 . The fourth tends to zero by (5.7). The last term can be shown to approach zero by using Schwarz' inequality and recalling (5.7) and (5.4). This establishes the lemma.

LEMMA 4. If for each $t$ in the interval $\left[t_{1}, t_{2}\right]$ the quadratic form

$$
c_{i j} v^{i} v^{j}
$$

is positive for all non-null vectors $v$ which satisfy the equations

$$
\phi_{r^{i}}^{\beta}\left(y_{0}(t), y_{0}^{\prime}(t)\right) v^{i}=0 \quad(\beta=1, \cdots, m),
$$

then inequality (7.2) is satisfied.

Let $E$ be the set of points $(t, v)$ in $(n+2)$-dimensional space satisfying the conditions $t_{1} \leqq t \leqq t_{2},|v|=1$. For each positive integer $p$ we define $U_{p}$ to be the set of all points $(t, v)$ at which

$$
c_{i j}(t) v^{i} v^{j}+p \sum_{\beta=1}^{m}\left(\phi_{r^{i}}^{\beta}\left(y_{0}(t), y_{0}^{\prime}(t)\right) v^{i}\right)^{2}>0 .
$$

Every point of $E$ which satisfies (7.6) is in $U_{p}$ for all $p$; every point of $E$ at which (7.6) is false is in $U_{p}$ if $p$ is large enough. By the Borel theorem a finite number of the $U_{p}$, say those with subscripts $p_{i}, \cdots, p_{k}$, cover $E$. Let $N$ be the greatest of these subscripts; then

$$
c_{i j}(t) v^{i} v^{i}+N \sum_{\beta=1}^{m}\left(\phi_{r i} v^{i}\right)^{2}>0
$$

for $t_{1} \leqq t \leqq t_{2}$ and $|v|=1$. By homogeneity, (7.8) continues to hold if $t_{1} \leqq t \leqq t_{2}$ and $|v| \neq 0$.

Now define

$$
I_{N}(\eta)=\int_{t_{1}}^{t_{2}}\left\{a_{i} \eta^{i} \eta^{j}+2 b_{i} \eta^{i} \dot{\eta}^{j}+c_{i} \dot{\eta}^{i} \dot{\eta}^{j}+N \sum_{\beta=1}^{m}\left(\phi_{y}^{\beta} \eta^{i}+\phi_{r i \eta^{j}}^{\beta}\right)^{2}\right\} d t
$$

The terms quadratic in $\dot{\eta}$ constitute the left member of (7.8), so $I_{N}$ satisfies the hypotheses of Lemma 3, and

$$
\lim \inf I_{N}\left(\eta_{q}\right) \geqq I_{N}\left(\eta_{0}\right) .
$$

Since $\eta_{0}$ satisfies the equations (3.6), the terms added to $I\left(\eta_{0}\right)$ in (7.9) vanish, so that

$$
I\left(\eta_{0}\right)=I_{N}\left(\eta_{0}\right)
$$

This is not true of $\eta_{q}$. But from (6.8), with (7.9) and (7.1), we find 


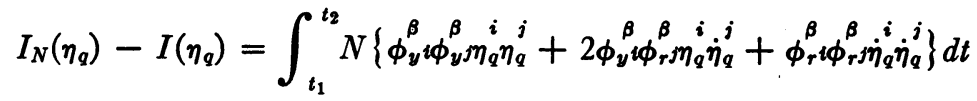

$$
\begin{aligned}
& =N \int_{t_{1}}^{t_{2}}\left\{\left[\phi_{y^{\prime} i \phi_{y^{j}}^{\beta}}^{\beta}-A_{q}^{\beta, i} A_{q}^{\beta, j}\right] \eta_{q}^{i} \eta_{q}^{j}\right. \\
& \left.+2\left[\phi_{y}^{\beta} i \phi_{r j}^{\beta}-A_{q}^{\beta, i} B_{q}^{\beta, j}\right] \eta_{q}^{i} \dot{\eta}_{q}^{j}+\left[\phi_{r^{\prime} i \phi_{r j}^{\beta}}^{\beta}-B_{q}^{\beta, i} B_{q}^{\beta, i}\right]{\dot{\eta_{q}}}_{q}^{i} \dot{\eta}_{q}^{j}\right\} d t .
\end{aligned}
$$

The quantities in square brackets tend uniformly to zero, by (6.10) and (6.11), and the integrals of the absolute values of their coefficients are bounded; so

$$
\lim _{q \rightarrow \infty}\left[I_{N}\left(\eta_{q}\right)-I\left(\eta_{q}\right)\right]=0
$$

From (7.10), (7.11) and (7.13) we obtain the conclusion (7.2) of our lemma.

LEMMA 5. If for each $t$ in the interval $\left[t_{1}, t_{2}\right]$ the quadratic form $c_{i j} v^{i} v^{j}$ is nonnegative whenever the vector $v$ is linearly independent of $y_{0}^{\prime}(t)$ and satisfies equations (7.6), then inequality (7.2) is satisfied.

Let $\epsilon$ be an arbitrary positive number, and let

$$
I_{\epsilon}(\eta)=\int_{t_{1}}^{t_{2}}\left[a_{i j} \eta^{i} \eta^{j}+2 b_{i j} \eta^{i} \dot{\eta}^{j}+c_{i j} \dot{\eta}^{i} \dot{\eta}^{j}+\epsilon \dot{\eta}^{i} \dot{\eta}^{i}\right] d t .
$$

The quadratic form $\dot{c}_{i j} v^{i} v^{j}$ is here replaced by

$$
c_{i j} v^{i} v^{j}+\epsilon v^{i} v^{i} .
$$

This is positive for all non-null vectors $v$ which satisfy equations (7.6). For the second term is positive, while the first is non-negative, by hypothesis if $v$ is linearly independent of $y_{0}^{\prime}$ and by continuity if $v$ is a multiple of $y_{0}^{\prime}$. So $I_{c}$ satisfies the hypotheses of Lemma 4 , and

$$
\lim I_{\epsilon}\left(\eta_{q}\right) \geqq I_{\epsilon}\left(\eta_{0}\right) \text {. }
$$

From (5.4) we deduce

$$
0 \leqq \int_{t_{1}}^{t_{2}} \dot{\eta}_{q}^{i} \dot{\eta}_{q}^{i} d t \leqq 1
$$

Hence

$$
I\left(\eta_{0}\right) \leqq I_{\epsilon}\left(\eta_{0}\right) \leqq \liminf _{q \rightarrow \infty} I_{\epsilon}\left(\eta_{q}\right) \leqq \lim _{q \rightarrow \infty} \inf I\left(\eta_{q}\right)+\epsilon .
$$

Since $\epsilon$ is an arbitrary positive number, this implies inequality (7.2), and the proof of the lemma is complete.

8. First case. We now distinguish two possible cases.

Case I. Either $u_{0} \neq(0, \cdots, 0)$ or else the functions $\eta_{0}^{i}(t)$ do not all vanish identically. 
Case II. The numbers $u_{0}^{h}$ are all 0 and the functions $\eta_{0}^{\imath}(t)$ all vanish identically.

In this section we shall discuss the first case.

Under the hypotheses of Case $\mathrm{I}$, the set $[\eta, u]$ is not essentially null. This is obvious from the definition if some $u^{h}$ is not zero. If all the $u^{h}$ have the value 0 , we have by (4.8) and (5.3)

$$
\eta_{q}^{i}(t) y_{0}^{i \prime}(t)=\left(A_{q} / k_{2}\right) t+\left(B_{q} / k_{q}\right) .
$$

The left member converges uniformly as $0 \rightarrow \infty$, so its limit $\eta_{0}^{1} y_{0}^{11}$ must be linear in $t$. But since $u_{0}=0$ equations (6.17) show that this linear function vanishes at $t_{1}$ and at $t_{2}$, so it is identically zero:

$$
\eta_{0}^{i}(t) y_{0}^{i \prime}(t) \equiv 0
$$$$
\left(t_{1} \leqq t \leqq t_{2}\right) \text {. }
$$

Now $\eta_{0}^{i}(t)$ cannot have the form $\rho(t) y_{0}^{y^{\prime}}(t)(i=0, \cdots, n)$; for then (8.2) would give $\rho \equiv 0$, contrary to the hypothesis that the $\eta_{0}^{8}$ do not all vanish identically.

By the hypotheses of Theorem I, there are multipliers $\lambda^{0} \geqq 0, \lambda^{1}(t), \cdots, \lambda^{m}(t)$ with which the set $\left(C_{0}, \alpha_{0}\right)$ satisfies the Euler equations, transversality condition and strengthened Clebsch condition and with which $J_{2}\left(\eta_{0}, u_{0}, \lambda\right)$ is positive. This last condition will not be used until the last paragraph of this section.

Since $\lambda^{0}$ is non-negative, inequality (4.6) implies

$$
\lambda^{0} J\left(C_{q}, \alpha_{q}\right)-\lambda^{0} J\left(C_{0}, \alpha_{0}\right) \leqq 0 .
$$

Recalling that along an admissible curve we have $F=\lambda^{0} f$, this can be written in the form

$$
\lambda^{0} \theta\left(\alpha_{q}\right)-\lambda^{0} \theta\left(\alpha_{0}\right)+\int_{t_{1}}^{t_{2}}\left\{F\left(y_{q}, \dot{y}_{q}, \lambda\right)-F\left(y_{0}, y_{0}^{\prime}, \lambda\right)\right\} d t \leqq 0 .
$$

By Taylor's theorem with integral form of remainder, this implies (with (5.2) and (5.3))

$$
\begin{aligned}
& \lambda^{0} \theta_{h}\left(\alpha_{0}\right) k_{q} u_{q}^{h}+k_{q}^{2} \lambda^{0} \int_{0}^{1}(1-\tau) \theta_{h k}\left(\alpha_{0}+\tau k_{q} u_{q}\right) u_{q}^{h} u_{q}^{k} d \tau \\
& +k_{q} \int_{t_{1}}^{t_{2}}\left\{F_{y^{i}}\left(y(t), y^{\prime}(t), \lambda(t)\right) \eta_{q}^{i}+F_{r} \dot{\eta}_{q}^{i}\right\} d t \\
& +k_{q}^{2} \int_{t_{1}}^{t_{2}} \int_{0}^{1}(1-\tau)\left\{F_{y^{i_{y} j}}\left(y+\tau k_{q} \eta_{q}, y^{\prime}+\tau k_{q} \dot{\eta}_{q}, \lambda\right) \eta_{q}^{i} \eta_{q}^{i}\right. \\
& \left.+2 F_{y^{i} r_{r} j \eta_{q} \dot{\eta}_{q}^{j}}+F_{r} i_{r} \dot{\eta}_{q}^{i} \dot{\eta}_{q}^{j}\right\} d \tau d t \leqq 0 .
\end{aligned}
$$

To the third term in (8.5) we apply the usual integration by parts. Since 
by hypothesis the Euler equations are satisfied, this term has the value

$$
\left.k_{q} F_{r i} \eta_{q}^{i}\right|_{t_{1}} ^{t_{2}}
$$

But

$$
\begin{aligned}
k_{q} \eta_{q}^{i}\left(t_{s}\right) & =y_{q}^{i}\left(t_{s}\right)-y_{0}^{i}\left(t_{s}\right) \\
& =T^{i s}\left(\alpha_{q}\right)-T^{i s}\left(\alpha_{0}\right) \\
& =k_{q} T_{h}^{i s}\left(\alpha_{0}\right) u_{q}^{h}+k_{q}^{2} \int_{0}^{1}(1-\tau) T_{h k}^{i s}\left(\alpha_{0}+\tau k_{q} u_{q}\right) u_{q}^{h} u_{q}^{k} d \tau
\end{aligned}
$$

We substitute this in (8.6), and substitute expression (8.6) for the third term in (8.5). Since by hypothesis the transversality condition is satisfied, inequality (8.5) yields, after division by $k_{q}^{2} / 2$,

$$
\begin{aligned}
D_{q} \equiv & 2 \int_{0}^{1}(1-\tau)\left\{\lambda^{0} \theta_{h k}\left(\alpha_{0}+\tau k_{q} u_{q}\right)\right. \\
& +F_{r i}\left(y_{0}\left(t_{2}\right), y_{0}^{\prime}\left(t_{2}\right), \lambda\left(t_{2}\right)\right) T_{h k}^{i 2}\left(\alpha_{0}+\tau k_{q} u_{q}\right) \\
& \left.-F_{r^{i}}\left(y_{0}\left(t_{1}\right), y_{0}^{\prime}\left(t_{1}\right), \lambda\left(t_{1}\right)\right) T_{h k}^{i 1}\left(\alpha_{0}+\tau k_{q} u_{q}\right)\right\} u_{q}^{h} u_{q}^{k} d \tau \\
& +2 \int_{t_{1}}^{t_{2}} \int_{0}^{1}(1-\tau)\left\{F_{y^{i} y^{j}}\left(y_{0}+\tau k_{q} \eta_{q}, y_{0}^{\prime}+\tau k_{q} \dot{\eta}_{q}, \lambda\right) \eta_{q}^{i} \eta_{q}^{j}\right. \\
& \left.+2 F_{y_{r}^{i} j \dot{\eta}_{q} \dot{\eta}_{q}^{j}}+F_{\left.r_{r} j \dot{\eta_{q}} \dot{\eta}_{q}^{i}\right\}}\right\} d \tau d t \leqq 0 .
\end{aligned}
$$

Therefore it is clear that

$$
\limsup _{q \rightarrow \infty} D_{q} \leqq 0
$$

Since by (5.3)

$$
y_{0}^{i}(t)+\tau k_{q} \eta_{0}^{i}(t)=y_{0}^{i}(t)+\tau\left[y_{q}^{i}(t)-y_{0}^{i}(t)\right],
$$

with a like equation for the derivatives, we see by (4.3) and (4.4) that

$$
\lim _{a \rightarrow \infty} F_{r i} j\left(y_{0}+\tau k_{q} \eta_{a}, y_{0}^{\prime}+\tau k_{q} \dot{\eta}_{q}, \lambda\right)=F_{r^{i} r^{j}}\left(y_{0}, y_{0}^{\prime}, \lambda\right)
$$

uniformly for $0 \leqq \tau \leqq 1$ and $t_{1} \leqq t \leqq t_{2}$. Relations similar to (8.10) hold for the other coefficients in (8.8). Hence if $\epsilon$ is an arbitrary positive number, for all sufficiently large values of $q$ the replacement of $k_{q}$ by 0 in (8.8) alters each coefficient by less than $\epsilon$. But after this replacement the variable $\tau$ has disappeared from (8.8) except in the factors $(1-\tau)$, whose integral from 0 to 1 has the value $1 / 2$. The result is that the left member of (8.8) takes the form $J_{2}\left(\eta_{q}, u_{q}, \lambda\right)$ (cf. (3.8)). Since each coefficient was changed by less than $\epsilon$, we thus find with the help of Schwarz' inequality 


$$
\begin{aligned}
\left|D_{q}-J_{2}\left(\eta_{q}, u_{q}, \lambda\right)\right| & \leqq \epsilon\left(\sum_{h=1}^{r} u_{q}^{h}\right)^{2}+\epsilon \int_{t_{1}}^{t_{2}}\left(\sum_{i=0}^{n}\left\{\left|\eta_{q}^{i}\right|+\left|\dot{\eta}_{q}^{i}\right|\right\}\right)^{2} d t \\
& \leqq \epsilon\left\{r\left|u_{q}\right|^{2}+2(n+1) \int_{t_{1}}^{t_{2}}\left(\left|\eta_{q}\right|^{2}+\left|\dot{\eta}_{q}\right|^{2}\right) d t\right\} .
\end{aligned}
$$

The coefficient of $\epsilon$ is bounded, since (5.4) is satisfied, and $\epsilon$ is arbitrary. Hence

$$
\lim _{q \rightarrow \infty}\left[D_{q}-J_{2}\left(\eta_{q}, u_{q}, \lambda\right)\right]=0 .
$$

Relations (8.9) and (8.12) imply

$$
\limsup _{q \rightarrow \infty} J_{2}\left(\eta_{q}, u_{q}, \lambda\right) \leqq 0 .
$$

By (5.5) we have

$$
\lim _{a \rightarrow \infty} b_{h k} u_{q}^{h} u_{q}^{k}=b_{h k} u_{0}^{h} u_{0}^{k} .
$$

The integral in (3.8) satisfies the hypotheses of Lemma 3, since the strengthened Clebsch condition holds. Hence from Lemma 3 and equation (8.14) we obtain

$$
\lim _{q \rightarrow \infty} \inf J_{2}\left(\eta_{q}, u_{q}, \lambda\right) \geqq J_{2}\left(\eta_{0}, u_{0}, \lambda\right) .
$$

But now we have reached our desired contradiction. For by the choice of the $\lambda^{0}$ and $\lambda^{\alpha}(t)$ the right member of (8.15) is positive, so inequalities (8.13) and (8.15) are incompatible.

9. Second case. We still have to dispose of Case II, in which the $u_{0}$ and $\eta_{0}(t)$ are all zero. The hypotheses of Theorem I do not mention such variation sets, so we must prove a lemma.

LEMMA 6. Under the hypotheses of Theorem I, there exist admissible variation sets which are not essentially null.

Bliss $\left({ }^{7}\right)$ has shown that there exist functions $\phi^{\gamma}(t, r)$ of class $C^{2}(\gamma=m$. $+1, \cdots, n+1)$ such that the determinant

$$
\left|\begin{array}{l}
\phi_{r^{i}}^{\beta}\left(y_{0}(t), y_{0}^{\prime}(t)\right) \\
\phi_{r^{i}}^{\gamma}\left(t, y_{0}^{\prime}(t)\right)
\end{array}\right|
$$

does not vanish on the interval $t_{1} \leqq t \leqq t_{2}$. In the interval we choose $n+3$ points $\tau_{l}$ such that

$$
t_{1} \leqq \tau_{1}<\tau_{2}<\cdots<\tau_{n+3} \leqq t_{2}
$$

(7) G. A. Bliss, The problem of Mayer with variable end points, these Transactions, vol. 19 (1918), pp. 305-314. 
For $l=1, \cdots, n+2$ we choose functions $\zeta_{l}^{k}(t)(k=1, \cdots, n+1)$ with the following properties. If $k=1, \cdots, m$, then $\zeta_{l}^{k}(t)$ is identically zero. Except on $\left[\tau_{l}, \tau_{l+1}\right]$ the other $\zeta_{l}^{k}(t)$ also vanish identically. On $\left[\tau_{l}, \tau_{l+1}\right]$ the $\zeta_{l}^{k}(t)$ are constants, and the vector

$$
\left(\zeta_{l}^{1}(t), \cdots, \zeta_{l}^{n+1}(t)\right)
$$

is linearly independent of

$$
\left(\phi_{r^{i}}^{1} y_{0}^{i \prime}\left(\tau_{l}\right), \cdots, \phi_{r^{i}}^{n+1} y_{0}^{i \prime}\left(\tau_{l}\right)\right) .
$$

This last condition can be satisfied since by hypothesis $m$ is less than $n$, so that we have the free choice of at least the last two components of the vector (9.2).

By known theorems on differential equations, for $l=1, \cdots, n+2$ the equations

$$
\begin{aligned}
\phi_{y^{i}}^{\beta}\left(y_{0}, y_{0}^{\prime}\right) H_{l}^{i}+\phi_{r^{i}}^{\beta}\left(y_{0}, y_{0}^{\prime}\right) H_{l}^{i \prime} & =0, \quad \phi_{r^{i}}^{\gamma}\left(t, y_{0}^{\prime}\right) H_{l}^{i \prime}=\zeta_{l}^{\gamma}(t) \\
(\beta & =1, \cdots, m ; \gamma=m+1, \cdots, n+1)
\end{aligned}
$$

have unique solutions $H_{l}^{i}$ vanishing at $t_{1}$. The $n+1$ homogeneous equations

$$
c_{l} H_{l}^{i}\left(t_{2}\right)=0 \quad(i=0,1, \cdots, n)
$$

in the $n+2$ unknowns $c_{l}$ have a non-trivial solution. We define

$$
\bar{\eta}^{i}(t)=c_{l} H_{l}^{i}(t) \quad\left(t_{1} \leqq t \leqq t_{2}\right) .
$$

Then $\bar{\eta}(t)$ satisfies the equations (3.6), because of (9.4). Since $\bar{\eta}$ vanishes at $t_{1}$ and at $t_{2}$, it satisfies (3.7) with $\bar{u}=0$. Hence $(\bar{\eta}, \bar{u})$ is an admissible variation set. If it were essentially null, there would be a function $\rho(t)$ such that

$$
\bar{\eta}^{i}(t)=\rho(t) y_{0}^{i \prime}(t)
$$$$
\left(t_{1} \leqq t \leqq t_{2}\right) \text {. }
$$

This $\rho(t)$ is easily seen to be continuous and to have corners only at the points $\tau_{l}$. If $\lambda$ is the least integer such that $c_{\lambda} \neq 0$, then $\bar{\eta}^{i}(t)$ is identically zero on $\left[t_{1}, \tau_{\lambda}\right]$. By (9.7) and (9.6)

$$
H_{\lambda}^{i \prime}\left(\tau_{\lambda}+\right)=\bar{\eta}^{i \prime}\left(\tau_{\lambda}+\right) / c_{\lambda}=\rho^{\prime}\left(\tau_{\lambda}+\right) y_{0}^{i \prime}\left(\tau_{\lambda}\right) / c_{\lambda} .
$$

Since $H_{\lambda}^{i}\left(t_{\lambda}\right)$ vanishes, by (9.4) we see that for $l=\lambda$ the vector (9.2) is a multiple of (9.3), contrary to its choice. Lemma 6 is therefore established.

Now by Lemma 6 and hypothesis (3) of Theorem I there are multipliers $\lambda^{0} \geqq 0, \lambda^{1}(t), \cdots, \lambda^{m}(t)$ with which the Euler equations, transversality condition and strengthened Clebsch condition hold. From the last mentioned condition we see that the form 


$$
F_{r^{i} r}\left(y_{0}(t), y_{0}^{\prime}(t), \lambda\right) v^{i_{0} j}
$$

is positive on the set of unit vectors $v$ which are orthogonal to $y_{0}^{\prime}$ and satisfy equations (3.5). This set of vectors is bounded and closed, so on it the form (9.8) has a positive lower bound, which we denote by $2 \epsilon$. It follows that on the set of vectors $v$ just described the inequality

$$
F_{r i r} j v^{i} v^{j}-\epsilon v^{i} v^{i}>0
$$

holds, since the coefficient of $\epsilon$ has the value 1. By homogeneity, (9.9) continues to hold all non-null vectors $v$ which are orthogonal to $y_{0}^{\prime}$ and satisfy equations (3.5).

Let $v$ be any vector which satisfies (3.5) and is linearly independent of $y_{0}^{\prime}$. It can be resolved into components

$$
\nu^{i}=v_{0}^{i}+\delta y_{0}^{i \prime}(t)
$$

where $v_{0}$ is orthogonal to $y_{0}^{\prime}$. Since $v$ is linearly independent of $y_{0}^{\prime}$, the component $v_{0}$ is not null. The homogeneity of $F$ and $\phi^{\beta}$ entails the well known consequence

$$
y_{0}^{i \prime}(t) F_{r} \imath_{r j}\left(y_{0}, y_{0}^{\prime}, \lambda\right)=0, \quad y_{0}^{i{ }^{\prime} \phi_{r^{i}}}=0 .
$$

Now $y_{0}^{\prime}$ and $v$ both satisfy the linear equations (3.5), hence by (9.10) $v_{0}$ also satisfies those equations. Therefore (9.9) holds with $v_{0}$ in place of $v$, and with the help of (9.11) we deduce

$$
F_{r i r}\left(y_{0}, y_{0}^{\prime}, \lambda\right) v^{i} v^{j}-\epsilon\left[v^{i} v^{i}-\left(y_{0}^{i \prime} v^{i}\right)^{2}\right]=F_{r i r} v_{0}^{i} v_{0}^{j}-\epsilon v_{0}^{i} v_{0}^{i}>0 .
$$

Inequality (9.12) shows that the integral

$$
I_{\epsilon}(\eta)=\int_{t_{1}}^{t_{2}}\left\{2 \omega(t, \eta, \dot{\eta})-\epsilon\left[\dot{\eta}^{i} \dot{\eta}^{i}-\left(y_{0}^{i} \dot{\eta}^{i}\right)^{2}\right]\right\} d t
$$

satisfies the hypotheses of Lemma 3, so that

$$
\lim \inf I_{\epsilon}\left(\eta_{q}\right) \geqq I_{\epsilon}\left(\eta_{0}\right)=0 .
$$

Since $u_{q}$ approaches $u_{0}=0$ as $q \rightarrow \infty$, this and (3.8) together imply

$$
\lim \inf \left\{J_{2}\left(\eta_{q}, u_{q}, \lambda\right)-\epsilon \int_{t_{1}}^{t_{2}}\left[\dot{\eta}_{q}^{i} \dot{\eta}_{q}^{i}-\left(y_{0}^{i_{1}^{\prime}} \dot{\eta}_{q}^{i}\right)^{2}\right] d t\right\} \geqq 0 .
$$

By (4.8) and (5.3) the function

$$
y_{0}^{i \prime}(t) \eta_{q}^{i}(t)
$$

is linear in $t$, and it converges uniformly to zero, since $\eta_{0}$ is zero. Except on a set of measure zero we have 


$$
y_{0}^{i \prime} \dot{\eta}_{q}^{i}=\left(\begin{array}{c}
y_{0}^{\prime \prime} \eta_{q}^{i}
\end{array}\right)^{\prime}-y_{0}^{i \prime \prime} \eta_{q}^{i},
$$

and both terms on the right tend uniformly to zero. So the last term in the square bracket in (9.15) can be omitted without affecting the limit. In (5.4) the first and second terms tend to zero, so the third term tends to 1 as $q \rightarrow \infty$. Thus (9.15) implies

$$
\lim \inf J_{2}\left(\eta_{q}, u_{q}, \lambda\right)-\epsilon \geqq 0 .
$$

On the other hand, the considerations leading, to inequality (8.13) are applicable to Case II as well to Case I, so inequality (8.13) must hold. This contradicts (9.16). Hence in each of the two possible cases we have arrived at a contradiction, and Theorem $I$ is established.

10. Statement of problem in non-parametric form. From Theorem I we can deduce its analogue for problems in non-parametric form. We use the formulation due to Morse and Myers $(8,9)$. The functions

$$
\begin{aligned}
f(x, z, p) & =f\left(x, z^{1}, \cdots, z^{n}, p^{1}, \cdots, p^{n}\right) \\
\phi^{\beta}(x, z, p) & =\phi^{\beta}\left(x, z^{1}, \cdots, z^{n}, p^{1}, \cdots, p^{n}\right) \quad(\beta=1, \cdots, m<n)
\end{aligned}
$$

will be supposed to be defined and of class $C^{2}$ on an open point set $S_{1}$ in $(x, z, p)$-space. The functions $T^{i s}(\alpha)(i=0, \cdots, n ; s=1,2)$ are defined and of class $C^{2}$ on an open set $R_{2}$ in $\left(\alpha^{1}, \cdots, \alpha^{r}\right)$-space. An admissible set $[z, \alpha]$ is a set of functions $z^{i}(x)$ absolutely continuous on an interval $\left[x_{1}, x_{2}\right]$ such that for almost all $x$ in $\left[x_{1}, x_{2}\right]$ the point $(x, z(x), \dot{z}(x))$ is in $S_{1}$ and satisfies the equations

$$
\phi^{\beta}(x, z(x), \dot{z}(x))=0 \quad(\beta=1, \cdots, m),
$$

together with a set of parameters $\alpha$ in $R_{2}$ with which the end conditions

$$
x_{s}=T^{0 s}(\alpha), \quad z^{c}\left(x_{s}\right)=T^{c s}(\alpha) \quad(c=1, \cdots, n ; s=1,2)
$$

are satisfied.

The problem of Bolza in non-parametric form is the problem of minimizing the functional

$$
J[z, \alpha] \equiv \theta(\alpha)+\int_{x_{1}}^{x_{2}} f\left(x, z, z^{\prime}\right) d x
$$

in the class of admissible sets $[z, \alpha]$.

Let $\left[z_{0}, \alpha_{0}\right]$ be an admissible set in which the functions

$$
z^{c}=z_{0}^{c}(x) \quad\left(x_{1,0} \leqq x \leqq x_{2,0} ; c=1,2, \cdots, n\right)
$$

(8) M. Morse and S. B. Myers, The problems of Lagrange and Mayer with variable end points, Proceedings of the American Academy of Arts and Sciences, vol. 66 (1931), pp. 235-253.

(9) M. R. Hestenes, Sufficient conditions for the problem of Bolza in the calculus of variations, these Transactions, vol. 36 (1934), pp. 793-818. 
are of class $C^{1}$. The set $\left[z_{0}, \alpha_{0}\right]$ satisfies the Euler equations with multipliers $\lambda^{0}, \lambda^{1}(x), \cdots, \lambda^{m}(x)$ if the functions

$$
F(x, z, p, \lambda) \equiv \lambda^{0} f(x, z, p)+\lambda^{\beta} \phi^{\beta}(x, z, p)
$$

satisfy the equations

$$
\frac{d}{d x} F_{p c}\left(x, z_{0}, z_{0}^{\prime}, \lambda\right)=F_{z}\left(x, z_{0}, z_{0}^{\prime}, \lambda\right) \quad(c=1, \cdots, n) .
$$

It satisfies the transversality conditions with multipliers $\lambda^{0}, \lambda^{1}(x), \cdots, \lambda^{m}(x)$ if

$$
\lambda^{0} \theta_{h}\left(\alpha_{0}\right)+\left[\left(F-z_{0}^{c \prime} F_{p c}\right) T_{h}^{0 s}+F_{p c} T_{h}^{c s}\right]_{1}^{2}=0 \quad(h=1, \cdots, r) .
$$

As usual, $c$ has the range $1, \cdots, n$. The square-bracketed symbol in (10.7) is to be understood as follows. The functions $F$, and so on are first evaluated at $\left(x_{s, 0}, z_{0}\left(x_{s, 0}\right), z_{0}^{\prime}\left(x_{s, 0}\right), \lambda\left(x_{s, 0}\right)\right), s=1,2$. Then the value of the sum inside the square bracket is evaluated for $s=1$ and for $s=2$, and the former value subtracted from the latter.

The set $\left[z_{0}, \alpha_{0}\right]$ satisfies the strengthened Clebsch condition with multipliers $\lambda^{0}, \lambda^{1}(x), \cdots, \lambda^{m}(x)$ if for each $x$ in $\left[x_{1,0}, x_{2,0}\right]$ the inequality

$$
F_{p c} d\left(x, z_{0}(x), z_{0}^{\prime}(x), \lambda(x)\right) v^{c} v^{d}>0
$$

(summed over $c, d=1, \cdots, n)$ holds for all sets of numbers $\left(v^{1}, \cdots, v^{n}\right)$ $\neq(0, \cdots, 0)$ satisfying the equations

$$
\phi_{p c}^{\beta}\left(x, z_{0}(x), z_{0}^{\prime}(x)\right) v^{c}=0 \quad(\beta=1, \cdots, m) .
$$

An admissible variation set $[\zeta(x), u]$ is a set consisting of $n$ functions, absolutely continuous and having derivatives whose squares are summable over $\left[x_{1,0}, x_{2,0}\right]$ and satisfying the equations

$$
\phi_{z^{c}}^{\beta}\left(x, z_{0}, z_{0}^{\prime}\right) \zeta^{c}(x)+\phi_{p c}^{\beta}\left(x, z_{0}, z_{0}^{\prime}\right) \dot{\zeta}^{c}(x)=0 \quad(\beta=1, \cdots, m)
$$

for almost all $x$, and a set of numbers $\left(u^{1}, \cdots, u^{r}\right)$ with which the equations

$$
\zeta^{c}\left(x_{s, 0}\right)=\left[T_{h}^{c s}\left(\alpha_{0}\right)-z_{0}^{c \prime}\left(x_{s, 0}\right) T_{h}^{0 s}\right] u^{h} \quad(s=1,2 ; h=1, \cdots, r)
$$

hold.

If $[\zeta, u]$ is an admissible variation set, we define the second variation due to $[\zeta, u]$ by the equation

$$
J_{2}[\zeta, u, \lambda]=b_{h k} u^{h} u^{k}+\int_{x_{1,0}}^{x_{2,0}} 2 \omega(x, \zeta, \zeta) d x
$$

where 


$$
\begin{aligned}
b_{h k}= & \lambda^{0} \theta_{h k}\left(\alpha_{0}\right)+\left[\left(F_{x}-z_{0}^{c \prime} F_{z c}\right) T_{h}^{08} T_{k}^{0 s}+\left(F-z_{0}^{c \prime} F_{p c}\right) T_{h k}^{0 s}\right. \\
& \left.+F_{z^{c}}\left(T_{h}^{08} T_{k}^{c s}+T_{k}^{0 s} T_{h}^{c s}\right)+F_{p c} T_{h k}^{c s}\right]_{1}^{2}
\end{aligned}
$$

and

$$
2 \omega(x, \zeta, \pi)=F_{z} c_{z} d\left(x, z_{0}, z_{0}^{\prime}, \lambda\right) \zeta^{c} \zeta^{d}+2 F_{z^{c} p^{d} \zeta^{c} \pi^{d}+F_{p} p^{d} \pi^{c} \pi^{d}}
$$

The concept of a weak relative minimum will be carried over unchanged from the parametric problem. Let the functions (10.4) be of class $C^{1}$, and consider another set of absolutely continuous functions

$$
z^{c}=z^{c}(x), \quad x_{1} \leqq x \leqq x_{2} .
$$

We can use these functions to define a curve $C$ in $(n+1)$-space by means of the equation

$$
x=t, \quad z^{c}=z^{c}(t) \quad\left(x_{1} \leqq t \leqq x_{2}, c=1, \cdots, n\right),
$$

and likewise for the set (10.4). For these curves the concept of first order $\epsilon$-neighborhood has already been defined in $\$ 2$, and so has the concept of weak relative minimum.

For problems in non-parametric form we shall establish the following analogue of Theorem I.

THEOREM II. Let the following hypotheses be satisfied.

(1) The set $\left[z_{0}, \alpha_{0}\right]$ defined by (10.4) is admissible, and the functions $z_{0}^{c}(x)$ $(c=1, \cdots, n)$ are of class $C^{2}$.

(2) For each $x$ in the interval $\left[x_{1,0}, x_{2,0}\right]$ the matrix

$$
\left\|\phi_{p c}^{\beta}\left(x, z_{0}(x), z_{0}^{\prime}(x)\right)\right\| \quad(\beta=1, \cdots, m ; c=1, \cdots, n)
$$

has rank $m$.

(3) To each nonidentically vanishing admissible variation set $[\zeta, u]$ there corresponds a set of absolutely continuous multipliers $\lambda^{0} \geqq 0, \lambda^{1}(x), \cdots, \lambda^{m}(x)$ with which the set $\left[z_{0}, \alpha_{0}\right]$ satisfies the Euler equations, transversality condition and strengthened Clebsch condition, and with which the inequality

$$
J_{2}[\zeta, u, \lambda]>0
$$

is satisfied.

Then the set $\left[z_{0}, \alpha_{0}\right]$ gives $J[z, \alpha]$ a proper weak relative minimum on the class of admissible sets $[z, \alpha]$.

In the next two sections we shall show that this theorem is in fact a consequence of Theorem I.

11. Transformation into parametric form. We prove Theorem II by replacing the non-parametric problem of $\$ 10$ by an equivalent parametric problem. The symbols $y^{0}, y^{1}, \cdots, y^{n}$ will be used as alternative names for the 
$x, z^{1}, \cdots, z^{n}$ axes in $(n+1)$-space. In the $(2 n+2)$-dimensional space of points $(y, r)=\left(y^{0}, \cdots, y^{n}, r^{0}, \cdots, r^{n}\right)$ we define $R_{1}$ to be the set of points $(y, r)$ having $r^{0}>0$ and such that $\left(y^{0}, y^{1}, \cdots, y^{n}, r^{1} / r^{0}, \cdots, r^{n} / r^{0}\right)$ is in $S_{1}$. On $R_{1}$ we define functions $g(y, r), \psi^{\beta}(y, r)$ by the equations

$$
\begin{aligned}
g(y, r) & =r^{0} f\left(y^{0}, y^{1}, \cdots, y^{n}, r^{1} / r^{0}, \cdots, r^{n} / r^{0}\right), \\
\psi^{\beta}(y, r) & =r^{0} \phi^{\beta}\left(y^{0}, y^{1}, \cdots, y^{n}, r^{1} / r^{0}, \cdots, r^{n} / r^{0}\right) .
\end{aligned}
$$

These have the continuity and homogeneity properties specified in $\$ 2$.

From (11.1) we deduce

$$
g\left(y^{0}, \cdots, y^{n}, 1, r^{1}, \cdots, r^{n}\right)=f\left(y^{0}, y^{n}, r^{1}, \cdots, r^{n}\right),
$$

from which by differentiation we obtain similar identities for all existing partial derivatives not involving differentiation with respect to $r^{\mathbf{0}}$; for instance,

$$
g_{y^{0}}\left(y^{0}, \cdots, y^{n}, 1, r^{1}, \cdots, r^{n}\right)=f_{x}\left(y^{0}, \cdots, y^{n}, r^{1}, \cdots, r^{n}\right)
$$

Moreover, identities analogous to (11.2) and its corollaries are also valid for each of the functions $\phi^{\beta}$.

We now have the data needed for setting up the parametric problem of minimizing the functional

$$
I(C, \alpha) \equiv \theta(\alpha)+\int_{t_{1}}^{t_{2}} g(y(t), \dot{y}(t)) d t
$$

in the class of sets $(C, \alpha)$ for which the curves $C: y=y(t)$ have $(y, \dot{y})$ in $R_{1}$ for almost all $t$, satisfy the differential equations

$$
\psi^{\beta}(y, \dot{y})=0
$$$$
(\beta=1, \cdots, m)
$$

for almost all $t$, and satisfy the end conditions

$$
y^{i}\left(t_{s}\right)=T^{i s}(\alpha) \quad(i=0,1, \cdots, n ; s=1,2) .
$$

From (11.1) it is evident that if the set $[z, \alpha]$ with functions $z(t)$ defined by (10.15) is admissible, and $C$ is defined by $(10.16)$, then $(C, \alpha)$ is admissible for the parametric problem, and

$$
I(C, \alpha)=J[z, \alpha] .
$$

(The converse also is true; if $(C, \alpha)$ is admissible for the parametric problem, $C$ can be represented in the form (10.16), and the functions $z^{c}(x)$ thus obtained are admissible with parameters $\alpha$ for the non-parametric problem. But we do not need this.) Theorem II will therefore be established if we can show that its hypotheses imply that the corresponding parametric problem satisfies the hypotheses of Theorem I.

12. Verification of the hypotheses of Theorem I. Hypotheses (1) and (2) of Theorem I follow at once from the corresponding hypotheses of Theorem II. 
Let $[\eta(t), u]$ be an admissible variation set for the parametric problem. We define

$$
\zeta^{i}(x)=\eta^{i}(x)-y_{0}^{i \prime}(x) \eta^{0}(x) \quad\left(i=0, \cdots, n_{\mathrm{i}} x_{1,0} \leqq x \leqq x_{2,0}\right) .
$$

(The notations $x$ and $t$ for the independent variable are interchangeable, because of (10.16); and for the same reason $z^{c}(x)$ and $y^{c}(x)$ are identical, $c=1,2, \cdots, n$.)

If we recall that

$$
y_{0}{ }^{\prime}(t) \equiv 1
$$

a well known consequence of the homogeneity of the $\psi^{\beta}$ implies (with (3.6) and the analogues of (11.1))

$$
\begin{aligned}
\phi_{z c}^{\beta}\left(x, z_{0}, z_{0}^{\prime}\right) \zeta^{c}+ & \phi_{p c}^{\beta}\left(x, z_{0}, z_{0}^{\prime}\right) \dot{\zeta}^{c} \\
& =\psi_{y^{\imath}}^{\beta}\left(y_{0}, y_{0}^{\prime}\right)\left(\eta^{i}-y_{0}^{i \prime} \eta^{0}\right)+\psi_{r^{\imath}}^{\beta}\left(\dot{\eta}^{i}-y_{0}^{i \prime \prime} \eta^{0}-y_{0}^{i \prime} \dot{\eta}^{0}\right) \\
& =-\eta^{0}\left[\psi_{y^{\imath}}^{\beta} y_{0}^{i \prime}+\psi_{r^{i} y_{0}^{\prime \prime \prime}}\right] \\
& =-\eta^{0}\left[\psi^{\beta}\left(y_{0}, y_{0}^{\prime}\right)\right]^{\prime} \\
& =0 .
\end{aligned}
$$

So equations (10.10) hold. Equations (10.11) follow at once from (12.1) and (3.7). Therefore, by hypothesis (3) of Theorem II there exist multipliers $\lambda^{0} \geqq 0, \lambda^{1}(x), \cdots, \lambda^{m}(x)$ with which the Euler equations, transversality condition and strengthened Clebsch condition hold for the non-parametric problem, and also the inequality (10.18).

Let us define

$$
G(y, r, \lambda)=\lambda^{0} g(y, r)+\lambda^{\beta} \psi^{\beta}(y, r) .
$$

The Euler equations for the non-parametric problem constitute the last $n$ of the $n+1$ Euler equations for the parametric problem. But from the homogeneity of $G$ it can be shown that the equation

$$
y^{i^{\prime}}(t)\left\{\frac{d}{d t} G_{r^{i}}\left(y, y^{\prime}, \lambda\right)-G_{y^{i}}\left(y, y^{\prime}, \lambda\right)\right\}=0
$$

holds for any admissible curve $y^{i}=y^{i}(t)$ of class $C^{2}$. Since (12.2) holds and the last $n$ of the factors in braces vanish for $y_{0}(t)$, the first also vanishes, and all $n+1$ Euler equations are satisfied.

From the homogeneity relation

$$
G(y, r, \lambda)=r^{i} G_{r^{i}}(y, r, \lambda),
$$

with (12.2) and (11.1), we find that

$$
G_{r 0}\left(y_{0}, y_{0}^{\prime}, \lambda\right)=F\left(x, z_{0}, z^{\prime}, \lambda\right)-z_{0}^{c} F_{p c}\left(x, z_{0}, z_{0}^{\prime}, \lambda\right) .
$$


This shows that the transversality conditions (10.7) imply the transversality conditions (3.3) for the parametric problem.

Let $\left(v^{0}, \cdots, v^{n}\right)$ be a vector linearly independent of $y_{0}^{\prime}(t)$ and satisfying the equations (3.5) with $\psi^{\beta}$ in place of $\phi^{\beta}$. Define

$$
w^{c}=v^{c}-v^{0} y_{0}^{c \prime}(t) \quad(c=1, \cdots, n) .
$$

By the analogue of (12.6) and (12.2),

$$
\begin{aligned}
\phi_{p c}^{\beta}\left(x, z_{0}, z_{0}^{\prime}\right) w^{c} & \left.=\psi_{r^{i}\left(\nu^{i}\right.}{ }^{i}-v^{0} y_{0}^{i \prime}\right) \\
& =\psi_{r^{\prime} i v^{i}}{ }^{i} \nu^{0} \psi^{\beta}\left(y_{0}, y_{0}^{\prime}\right) \\
& =0 .
\end{aligned}
$$

Also, the $w^{c}$ are not all zero, since $\left(v^{0}, \cdots, v^{n}\right)$ is not a multiple of $y_{0}^{\prime}(t)$. Since by hypothesis the strengthened Clebsch condition holds,

$$
F_{p^{c} p^{d}}\left(x, z_{0}, z_{0}^{\prime}, \lambda\right) w^{c} w^{d}>0 .
$$

A consequence of the homogeneity of $G$ is

$$
G_{r^{i} r^{j}}(y, r, \lambda) r^{j}=0 .
$$

From this and (12.8) we have

$$
G_{r r_{r}}\left(y_{0}, y_{0}^{\prime}, \lambda\right) v^{i} v^{j}=F_{p c} d\left(x, z_{0}, z_{0}^{\prime}, \lambda\right) w^{c} w^{d},
$$

which is positive by (12.9). Therefore the strengthened Clebsch condition holds for the parametric problem.

Let us define

$$
2 \omega^{*}(t, \eta, \rho) \equiv G_{y} i_{y} j\left(y_{0}, y_{0}^{\prime}, \lambda\right) \eta^{i} \eta^{i}+2 G_{y^{i} r} \eta^{i} \rho^{i}+G_{r} i_{r} j \rho^{i} \rho^{j}
$$

This is a symmetric quadratic form in $(\eta, \rho)$, whence

$$
2 \omega^{*}(t, \eta, \rho)=\eta^{i} \omega_{\eta}^{*}(t, \eta, \rho)+\rho^{i} \omega_{\rho i}^{*}(t, \eta, \rho)
$$

and

$$
\bar{\eta}^{i} \omega_{\eta^{i}}^{*}(t, \eta, \rho)+\bar{\rho}^{i} \omega_{\rho i}^{*}(t, \eta, \rho)=\eta^{i} \omega_{\eta}^{*}(t, \bar{\eta}, \bar{\rho})+\rho^{i} \omega_{\rho i}^{*}(t, \bar{\eta}, \bar{\rho}) .
$$

(These.identities can also be established easily by direct computation.) It is well known that for every set of functions $\eta^{i}(t)$ of class $C^{2}$ the equations

$$
y_{0}^{i \prime}(t)\left\{\frac{d}{d t} \omega_{\rho i}^{*}\left(t, \eta, \eta^{\prime}\right)-\omega_{\eta^{i}}^{*}\left(t, \eta, \eta^{\prime}\right)\right\}=0
$$

are satisfied identically. We now prove a lemma.

LEMMA 7. If the functions $\gamma(t), \eta^{0}(t), \therefore, \eta^{n}(t)$ are absolutely continuous and the squares of their derivatives are summable, then 


$$
\begin{aligned}
\int_{t_{1}}^{t_{2}}\left\{\left(\gamma y_{0}^{i \prime}\right) \cdot \omega_{\rho l}^{*}(t, \eta, \dot{\eta})+\left(\gamma y_{0}^{i \prime}\right) \omega_{\eta^{l}}^{*}\left(t, \eta, \eta^{\prime}\right)\right\} d t \\
=\left.\gamma(t) \eta^{i}(t) G_{y^{t}}\left(y_{0}(t), y_{0}^{\prime}(t), \lambda(t)\right)\right|_{t_{1}} ^{t_{2}} .
\end{aligned}
$$

Let us first suppose that the $\eta^{i}(t)$ are of class $C^{2}$. By an integration by parts, with use of (12.15), the left member of $(12.16)$ is reduced to

$$
\left.\gamma y_{0}^{i} \omega_{\rho}^{*}\right|_{t_{1}} ^{t_{2}}
$$

The analogue of (12.6) holds for $G_{y^{i}}$, and with the help of this and (12.10) the expression (12.17) transforms into the right member of (12.16). Hence the lemma holds if the $\eta^{i}(t)$ are of class $C^{2}$.

By hypothesis, the derivatives of the $\eta^{i}$ are of class $L^{(2)}$, so by a known property of the Lebesgue integral there is a sequence of polynomials

such that

$$
p_{q}^{i \prime}(t) \quad(i=0,1, \cdots, u ; q=1,2, \cdots)
$$

$$
\lim _{a \rightarrow \infty} \int_{t_{1}}^{t_{2}}\left|\dot{\eta}-p_{q}^{\prime}\right|^{2} d t=0 .
$$

By the inequality of Schwarz, the functions

$$
p_{q}^{i}(t) \equiv \eta^{i}\left(t_{1}\right)+\int_{t_{1}}^{t} p_{q}^{i \prime}(t) d t
$$

converge uniformly to $\eta^{i}(t)$. The coefficients of the form $\omega^{*}$ are continuous, so

$$
\begin{aligned}
\lim _{q \rightarrow \infty} \int_{t_{1}}^{t_{2}} \sum_{i=0}^{n}\left\{\left[\omega_{\eta^{i}}^{*}\left(t, p_{q}, p_{q}^{\prime}\right)\right.\right. & \left.-\omega_{\eta^{\imath}}^{*}(t, \eta, \dot{\eta})\right]^{2} \\
& \left.+\left[\omega_{p^{t}}^{*}\left(t, p_{q}, p_{q}^{\prime}\right)-\omega_{p^{\imath}}^{*}(t, \eta, \dot{\eta})\right]^{2}\right\} d t=0 .
\end{aligned}
$$

For each $p_{q}$ the analogue of (12.16) holds, so by Schwarz' inequality

$$
\begin{aligned}
\int_{t_{1}}^{t_{2}}\left\{\left(\gamma y_{0}^{i \prime}\right)\right. & \left.\cdot \omega_{\rho}^{*}(t, \eta, \dot{\eta})+\left(\gamma y_{0}^{i \prime}\right) \omega_{\eta}^{*}(t, \eta, \dot{\eta})\right\} d t \\
& =\lim _{q \rightarrow \infty} \int_{t_{1}}^{t_{2}}\left\{\left(\gamma y_{0}^{i \prime}\right) \cdot \omega_{\rho^{\imath}}^{*}\left(t, p_{q}, p_{q}^{\prime}\right)+\left(\gamma y_{0}^{i \prime}\right) \omega_{\eta^{\imath}}^{*}\left(t, p_{q}, p_{q}^{\prime}\right)\right\} d t \\
& =\left.\lim _{q \rightarrow \infty} \gamma p_{q}^{i} G_{y^{\imath}}\left(y_{0}, y_{0}^{\prime}, \lambda\right)\right|_{t_{1}} ^{t_{2}} \\
& =\left.\gamma \eta^{i} G_{y^{\imath}}\left(y_{0}, y_{0}^{\prime}, \lambda\right)\right|_{t_{1}} ^{t_{2}} .
\end{aligned}
$$

This establishes the lemma. 
By definition (3.8),

$$
I_{2}(\eta, u, \lambda)=\lambda^{0} b_{k k}^{*} u^{h} u^{k}+\int_{t_{1}}^{t_{2}} 2 \omega^{*}(t, \eta, \dot{\eta}) d t
$$

where

$$
b_{h k}^{*}=\lambda^{0} \theta_{h k}+\left[G_{r i}\left(t_{s}\right) T_{h k}^{i s}\left(\alpha_{0}\right)\right]_{1}^{2} .
$$

From (12.13), (12.21) and (12.1) we deduce

$$
\begin{aligned}
& I_{2}(\eta, u, \lambda)=\lambda^{0} b_{h k}^{*} u^{h} u^{k}+\int_{t_{1}}^{t_{2}}\left\{\zeta^{i} \omega_{\eta^{i}}^{*}(t, \zeta, \zeta)+\dot{\zeta}^{i} \omega_{\rho}^{*}(t, \zeta, \dot{\zeta})\right\} d t \\
& +\int_{t_{1}}^{t_{2}}\left\{\zeta^{i} \omega_{\eta^{i}}^{*}\left(t, \eta^{0} y_{0}^{\prime},\left(\eta^{0} y_{0}^{\prime}\right)^{\prime}\right)+\dot{\zeta}^{i} \omega_{\rho}^{*} t\left(t, \eta^{0} y_{0}^{\prime},\left(\eta^{0} y_{0}^{\prime}\right)^{\prime}\right)\right\} d t \\
& +\int_{t_{1}}^{t_{2}}\left\{\eta^{0} y_{0}^{i \prime} \omega_{\eta}^{*}(t, \zeta, \zeta)+\left(\eta^{0} y_{0}^{i \prime}\right)^{\prime} \omega_{\rho^{i}}^{*}(t, \zeta, \dot{\zeta})\right\} d t \\
& +\int_{t_{1}}^{t_{2}}\left\{\eta^{0} y_{0}^{i_{1}} \omega_{\eta}^{*}\left(t, \eta^{0} y_{0}^{\prime},\left(\eta^{0} y_{0}^{\prime}\right)^{\prime}\right)\right. \\
& \left.+\left(\eta^{0} y_{0}^{i \prime}\right)^{\prime} \omega_{\rho i}^{*}\left(t, \eta^{0} y_{0}^{\prime},\left(\eta^{0} y_{0}^{\prime}\right)^{\prime}\right)\right\} d t .
\end{aligned}
$$

The integrand in the first integral in the right member is $2 \omega^{*}(t, \zeta, \dot{\zeta})$, which by $(12.12)$ is the same as $2 \omega(t, \zeta, \dot{\zeta})$, since $\zeta^{0}$ vanishes identically. By Lemma 7 , the third integral has the value

$$
\left.\eta^{0} \zeta^{i} G_{y^{\imath}}\left(y_{0}, y_{0}^{\prime}, \lambda\right)\right|_{t_{1}} ^{t_{2}} .
$$

Since $\zeta^{0}=0$, by (12.4) and (11.1) this is equal to

$$
\left.\eta^{0} \zeta^{c} F_{z c}\left(x, z_{0}, z_{0}^{\prime}, \lambda\right)\right|_{x_{1,0}} ^{x_{2,0}} .
$$

By the same argument, the fourth integral has the value

$$
\left.\eta^{0} \eta^{0} y_{0}^{i \prime} G_{y^{i}}\left(y_{0}, y_{0}^{\prime}, \lambda\right)\right|_{x_{1,0}} ^{x_{2,0}} \text {. }
$$

By (12.14), the second integral has the same value (12.25) as the third. We substitute these evaluations in (12.23), and for the end values of $\eta^{0}$ and $\zeta^{c}$ we substitute the values given by (3.7) and (10.11). On collecting terms and recalling (12.7), (10.12) and (10.13), we find

$$
I_{2}(\eta, u, \lambda)=J_{2}[\zeta, u, \lambda],
$$

which is positive by hypothesis. 
We have now verified all the hypotheses of Theorem I for the parametric formulation of our problem, so by that theorem the set $\left(C_{0}, \alpha_{0}\right)$ gives a proper weak relative minimum to $J(C, \alpha)$ on the class of admissible sets $(C, \alpha)$. This immediately implies that $\left[z_{0}, \alpha_{0}\right]$ gives $J[z, \alpha]$ a proper weak relative minimum on the class of admissible sets $[z, \alpha]$, and Theorem II is established.

13. A corollary. If it were not for our unusually inclusive definition of admissible variation set, Theorem II would at once include Hestenes' sufficiency theorem for weak relative minima $\left({ }^{10}\right)$. For Hestenes assumes the hypotheses of Theorem II, with the additional requirement that the multipliers can be chosen independently of the sets $[\zeta, u]$. However, it requires some proof to show that in this case the assumption that the second variation is positive for all variation sets admissible in the sense of $\$ 3$ is necessarily satisfied if the second variation is positive whenever $[\zeta, u]$ is an admissible set and the $\zeta^{c}(x)$ are of class $D^{1}$. We establish this for the normal case; as Bliss has shown( $\left.{ }^{11}\right)$, the theorem of Hestenes can be deduced from the sufficiency theorem for the normal problem.

For each real number $a$, let us define

$$
Q_{a}[\zeta, u, \lambda]=\lambda^{0} b_{h k} u^{h} u^{k}+a u^{h} u^{h}+\int_{x_{1,0}}^{x_{2,0}}\left\{2 \omega(x, \zeta, \dot{\zeta})+a \zeta^{c} \zeta^{c}\right\} d x .
$$

\section{Clearly}

$$
Q_{0}[\zeta, u, \lambda]=J_{2}[\zeta, u, \lambda] .
$$

As in the discussion of (7.7), we can show that if $a$ is sufficiently large the quadratic form

$$
\lambda^{0} b_{h k} u^{h} u^{k}+a u^{h} u^{h}
$$

is positive definite and

$$
2 \omega(x, \zeta, v)+a \zeta^{c} \zeta^{c}
$$

is positive for all nonidentically zero sets $(\zeta, v)$ satisfying (10.9). We choose such an $a$; then there is a positive $\epsilon$ such that

$$
\lambda^{0} b_{h k} u^{h} u^{k}+a u^{h} u^{h} \geqq \epsilon u^{h} u^{h}
$$

and

$$
2 \omega(x, \zeta, \dot{\zeta})+a \zeta^{c} \zeta^{c} \geqq \epsilon\left[\zeta^{c} \zeta^{c}+\dot{\zeta}^{c} \zeta^{c}\right]
$$

whenever $\dot{\zeta}$ satisfies (10.9).

Let $K_{1}$ be the collection of admissible sets $[\zeta, u]$ such that

(10) Hestenes, loc. cit., p. 816.

(11) G. A. Bliss, Normality and abnormality in the calculus of variations, these Transactions, vol. 43 (1938), pp. 365-376. 


$$
u^{h} u^{h}+\int_{x_{1,0}}^{x_{2,0}} \zeta^{c} \zeta^{c} d x=1 .
$$

For any $[\zeta, u]$ in $K_{1}$ and any number $b$ we have

$$
Q_{a}[\zeta, u, \lambda]-Q_{b}[\zeta, u, \lambda]=a-b .
$$

Since the forms (13.3) and (13.4) are non-negative, $Q_{a}$ has a non-negative lower bound $m$ on the class $K_{1}$. Let $\left[\zeta_{q}, u_{q}\right]$ be a sequence of sets in $K_{1}$ for which $Q_{a}$ tends to its lower bound $m$ on $K_{1}$. By (13.5) and (13.6),

$$
Q_{a}[\zeta, u, \lambda] \geqq \epsilon\left\{u^{h} u^{h}+\int_{x_{1,0}}^{x_{2,0}}\left(|\zeta|^{2}+|\zeta|^{2}\right) d x\right\},
$$

so the value of the expression in braces is bounded on the sequence $\left[\zeta_{q}, u_{q}\right]$. The boundedness of the integral of $\left|\dot{\zeta}_{q}\right|^{2}$ implies the equi-continuity of the $\zeta_{q}$, as in (5.6). The boundedness of $\left|u_{q}\right|$ implies the boundedness of $\left|\zeta_{q}\left(x_{1,0}\right)\right|$, and this with the equi-continuity of the $\zeta_{q}$ implies their uniform boundedness. By Ascoli's theorem, we can select a subsequence converging uniformly to a limit $\zeta_{0}(x)$; we suppose $\left[\zeta_{q}, u_{q}\right]$ such a sequence. We may also suppose that the $u_{q}$ converge to a limit $u_{0}$. As in $\S 5$, the $\zeta_{0}^{c}$ are absolutely continuous, and the squares of their derivatives are summable, and by Lemma 2

$$
Q_{a}\left[\zeta_{0}, u_{0}, \lambda\right] \leqq \liminf _{q \rightarrow \infty} Q_{a}\left[\zeta_{q}, u_{q}, \lambda\right]=m
$$

But $\left[\zeta_{0}, u_{0}\right]$ also belongs to $K_{1}$, so inequality is impossible in (13.10). That is. $\left[\zeta_{0}, u_{0}\right]$ minimizes $Q_{a}$ on the class $K_{1}$.

By (13.8), $\left[\zeta_{0}, u_{0}\right]$ also minimizes $Q_{a-m}$ on $K_{1}$, and

$$
Q_{a-m}\left[\zeta_{0}, u_{0}, \lambda\right]=0 \text {. }
$$

Since $Q_{a-m}$ is homogeneous of degree 2 in $[\zeta, \dot{\zeta}, u]$ we see that $Q_{a-m}$ is nonnegative for all admissible variation sets, and on this class $\left[\zeta_{0}, u_{0}\right]$ minimizes $Q_{a-m}$.

Now we need only a slight extension of Bliss' $\left.{ }^{(12}\right)$ proof of the multiplier rule to show that there are absolutely continuous multipliers $\mu^{0}, \mu^{1}(x), \cdots, \mu^{m}(x)$ such that for the function

$$
2 \Omega(x, \zeta, \pi, \mu)=2 \mu^{0} \omega(x, \eta, \pi)+\mu^{\beta}\left[\phi_{z}^{\beta} c\left(x, z_{0}, z_{0}^{\prime}\right) \zeta^{c}+\phi_{p c \pi}^{\beta}\right]
$$

the du Bois-Reymond relation is satisfied:

$$
\Omega_{x c}\left(x, \zeta_{0}, \dot{\zeta}_{0}\right)=a_{c}+\int_{x_{0,1}}^{x} \Omega_{\zeta c}\left(x, \zeta_{0}, \dot{\zeta}_{0}\right) d x
$$

(12) G. A. Bliss, The problem of Lagrange in the calculus of variations, American Journal of Mathematics, vol. 52 (1930), pp. 673-744. 
where $a_{c}$ is a constant $(c=1, \cdots, n)$. But if the minimizing curve is normal, so is $\left[\zeta_{0}, u_{0}\right]$. So we may suppose $\mu_{0}=1$. It follows that

$$
\Omega_{\pi^{c} c^{d}}\left(x, \zeta_{0}, \dot{\zeta}_{0}\right) v^{c} v^{d}=F_{p c p d}\left(x, z_{0}, z_{0}^{\prime}\right) v^{c} v^{d},
$$

and this last is positive for all nonzero sets $\left(v^{1}, \cdots, v^{n}\right)$ satisfying (10.9). From this we deduce without trouble that $\dot{\zeta}_{0}$ must be continuous, so that $\zeta_{0}^{c}$ is of class $C^{1}$.

Now by (13.2) and (13.8) we have

$$
J_{2}\left[\zeta_{0}, u_{0}, \lambda\right]=Q_{a-m}\left[\zeta_{0}, u_{0}, \lambda\right]-a+m=m-a .
$$

But since $\zeta_{0}$ is of class $C^{1}$, by hypothesis the left member is positive. Hence $m-a$ is positive, and the minimum of $J_{2}$ on the class $K_{1}$ is positive. It follows by homogeneity that $J_{2}$ is positive for all nonidentically vanishing admissible variation sets $[\eta, u]$, as was to be proved.

Since the proofs in this section are rather long, the following remark may not be amiss. Most of the theory of the calculus of variations, originally developed for functions of class $D^{1}$, can be extended without difficulty to absolutely continuous functions. It seems reasonable to suppose that in the majority of specific problems in which the inequality $J_{2}[\zeta, u, \lambda]>0$ can be established for functions $\zeta$ of class $D^{1}$, it will be possible to use essentially the same proof to establish the positiveness of $J_{2}$ for all variation sets admissible in the sense of $\$ 3$.

14. An example. We now exhibit an example of a problem to which Theorem II applies, but which is not covered by any previously published theorem. A simpler example could be given, but this one will have the virtue of being non-trivial. It is convenient to use subscripts instead of superscripts for enumeration of variables, and so on, since we need to use exponents.

Interior to the interval $[0,1]$ we choose three disjoint closed intervals $\delta_{1}, \delta_{2}, \delta_{3}$ all of the same length $\epsilon$ (necessarily less than $1 / 3$ ). It is easy to find functions $\gamma_{1}(x), \gamma_{2}(x)$ of class $C^{2}$ on $[0,1]$ such that $\gamma_{1}$ has the value 1 on $\delta_{1}$ and the value 0 on $\delta_{2}$ and $\delta_{3}$, while $\gamma_{2}$ has the value 1 on $\delta_{2}$ and the value 0 on $\delta_{1}$ and $\delta_{3}$. We define

$$
f\left(x, z_{1}, \cdots, z_{5}, p_{1}, \cdots, p_{5}\right) \equiv p_{1}^{2}+p_{2}^{2}+p_{3}^{2}-4 z_{1} p_{1}-4 z_{2} p_{2} .
$$

The problem is to minimize

$$
J[z, \alpha] \equiv \int_{0}^{1} f\left(x, z, z^{\prime}\right) d x
$$

in the class of sets $\left[z_{1}, \cdots, z_{5}, \alpha_{1}, \alpha_{2}, \alpha_{3}\right]$ with absolutely continuous functions $z_{i}(x)(i=1, \cdots, 5)$ which satisfy the end conditions

$$
\begin{aligned}
x_{1} & =z_{i}\left(x_{1}\right)=0 \quad(i=1, \cdots, 5), \\
x_{2} & =1, \quad z_{4}\left(x_{2}\right)=z_{5}\left(x_{2}\right)=0, \quad z_{3}\left(x_{2}\right)=\alpha_{3},
\end{aligned}
$$


and the differential equations

$$
\begin{aligned}
& \phi_{1}\left(x, z, z^{\prime}\right) \equiv z_{4}^{\prime}+z_{1} z_{1}^{\prime}-z_{2} z_{2}^{\prime}+\gamma_{1}(x) z_{3}^{\prime 3}=0, \\
& \phi_{2}\left(x, z, z^{\prime}\right) \equiv z_{5}^{\prime}+z_{1} z_{2}^{\prime}+z_{2} z_{1}^{\prime}+\gamma_{2}(x) z_{3}^{\prime 3}=0 .
\end{aligned}
$$

By use of Theorem II we shall show that the set

$$
z_{i}(x) \equiv 0, \quad \alpha_{1}=\alpha_{2}=\alpha_{3}=0
$$

gives $J[z, \alpha]$ a weak relative minimum on the class of admissible sets $[z, \alpha]$.

As usual, we define $F=\lambda_{0} f+\lambda_{1}(x) \phi_{1}+\lambda_{2}(x) \phi_{2}$. The Euler equations simplify to the form

$$
\lambda_{1}^{\prime}=\lambda_{2}^{\prime}=0,
$$

and therefore are satisfied for all sets of constant multipliers. Henceforth we assume $\lambda_{1}$ and $\lambda_{2}$ constant. The transversality condition is identically satisfied. The quadratic form (10.8) is

$$
2 \lambda_{0}\left(v_{1}^{2}+v_{2}^{2}+v_{3}^{2}\right)
$$

while equations (10.9) become

$$
\nu_{4}=v_{5}=0
$$

Hence the strengthened Clebsch condition holds if and only if $\lambda_{0}$ is positive.

Equations (10.10) take the form

$$
\dot{\zeta}_{4}=0, \quad \dot{\zeta}_{5}=0,
$$

and are therefore satisfied by any set $\left(\zeta_{1}, \cdots, \zeta_{5}\right)$ with $\zeta_{4}$ and $\zeta_{5}$ constant. Equations (10.11) become

$$
\begin{array}{ccc}
\zeta_{i}(0)=0 & (i=1, \cdots, 5), \\
\zeta_{1}(1)=u_{1}, \quad \zeta_{2}(1)=u_{2}, \quad \zeta_{3}(1)=u_{3}, & \zeta_{4}(1)=\zeta_{5}(1)=0 .
\end{array}
$$

Thus an admissible variation set is a set $[\zeta, u]$ in which $\zeta_{4}$ and $\zeta_{5}$ vanish identically and the other three $\zeta_{i}$ vanish at $x=0$ and are absolutely continuous and have derivatives summable with their squares, and in which the $u_{h}$ satisfy (14.7).

The coefficients $b_{h k}$ are all zero, so if we observe that certain of the terms in $2 \omega$ are perfect differentials and make use of (14.7) we obtain

$$
\begin{array}{r}
J_{2}[\zeta, u]=2 \lambda_{0} \int_{0}^{1}\left(\dot{\zeta}_{1}^{2}+\dot{\zeta}_{2}^{2}+\dot{\zeta}_{3}^{2}\right) d x-4 \lambda_{0}\left(u_{1}^{2}+u_{2}^{2}\right)+\lambda_{1}\left(u_{1}^{2}-u_{2}^{2}\right) \\
+2 \lambda_{2} u_{1} u_{2} .
\end{array}
$$

If for every nonidentically null admissible variation set we can choose constants $\lambda_{0}>0, \lambda_{1}$ and $\lambda_{2}$ for which this is positive, all the hypotheses of Theorem II will be verified. 
If the derivatives $\zeta_{i}$ vanish almost everywhere, the $\zeta_{i}$ are constants. In this case, by (14.7) the $\zeta_{i}$ vanish identically and the $u_{i}$ also vanish, so $[\zeta, u]$ is identically null. Therefore if $[\zeta, u]$ is not identically null, the integral in (14.8) is positive. If $u_{1}=u_{2}=0$, the choice $\lambda_{0}=1, \lambda_{1}=\lambda_{2}=0$ serves. Otherwise we could choose $\lambda_{0}=u_{1}^{2}+u_{2}^{2}, \lambda_{1}=4\left(u_{1}^{2}-u_{2}^{2}\right), \lambda_{2}=8 u_{1} u_{2}$. Then

$$
J_{2}[\zeta, u]=2\left(u_{1}^{2}+u_{2}^{2}\right) \int_{t_{1}}^{t_{2}}\left(\dot{\zeta}_{1}^{2}+\dot{\zeta}_{2}^{2}+\dot{\zeta}_{3}^{2}\right) d x>0 .
$$

It is easy to show $\left.{ }^{13}\right)$ that it is not possible to choose any one set of multipliers with which the second variation is positive for every nonidentically zero admissible variation set $[\zeta, u]$.

To show that the problem is not a trivial one, in which the extremal (14.5) is isolated, and also that the problem does not impose any hidden end conditions, let us choose any three numbers $\alpha_{1}, \alpha_{2}, \alpha_{3}$. Let $z_{1}, z_{2}$ be any Lipschitzian functions vanishing at $x=0$ and assuming the respective values $\alpha_{1}, \alpha_{2}$ at $x=1$. We determine three numbers, $a_{1}, a_{2}, a_{3}$ by the conditions

$$
\begin{aligned}
\epsilon a_{1}^{3}+(1 / 2)\left(\alpha_{1}^{2}-\alpha_{2}^{2}\right) & =0, \\
\epsilon a_{2}^{3}+\alpha_{1} \alpha_{2} & =0, \\
\epsilon\left(a_{1}+a_{2}+a_{3}\right)-\alpha_{3} & =0 .
\end{aligned}
$$

(The number $\epsilon$ was defined in the second paragraph of this section.) Let $\dot{z}_{3}$ be the function which has the value $a_{i}$ on the interval $\delta_{i}(i=1,2,3)$ and is zero elsewhere, and let

$$
z_{3}(x)=\int_{0}^{1} \dot{z}_{3}(x) d x
$$

By the last of equations (14.9) we have $z_{3}(1)=\alpha_{3}$. The functions $z_{4}, z_{5}$ are determined by (14.4), with the initial values 0 . If we integrate from 0 to 1 in (14.4), by (14.9) we find $z_{4}(1)=z_{5}(1)=0$. Hence the functions $z_{i}(x)$ satisfy the conditions (14.3) and (14.4). Furthermore, it is clear that they can be made to lie in an arbitrarily small first order neighborhood of (14.5) by restricting $\left|\dot{z}_{1}\right|$ and $\left|\dot{z}_{2}\right|$ to be uniformly small and restricting $\alpha_{1}, \alpha_{2}$ and $\alpha_{3}$ to lie near zero.

15. Extension to rectifiable curves. The proofs of our sufficiency theorems did not depend in any essential way on the continuity of the derivatives $y_{0}^{\prime}(t)$ or $z_{0}^{\prime}(x)$. Theorem I, for example, can be generalized by letting $C_{0}$ be a rectifiable curve. This of course requires an investigation of the concept of first order neighborhood in the space of rectifiable curves. Such an investiga-

(13) E. J. McShane, On the second variation in certain normal problems of the calculus, of variations, American Journal of Mathematics, vol. 63 (1941), $\$ 5$. 
tion has already been made(14). Also the formulation of the strengthened Clebsch condition must be altered. The condition as stated in $\S 3$ is equivalent, when the functions $y_{0}(t)$ are of class $C^{1}$, to the following.

There is a positive number $\epsilon$ such that the inequality

$$
F_{r^{i} r^{j}}\left(y_{0}(t), y_{0}^{\prime}(t), \lambda\right) v^{i} v^{i} \geqq \epsilon|v|^{2}
$$

holds for all $t$ and all vectors $v$ orthogonal to $y_{0}^{\prime}(t)$.

It is this latter form which seems appropriate for extension to the case of rectifiable curves. We say that a rectifiable curve $C_{0}: y^{t}=y_{0}^{i}(t), t_{1} \leqq t \leqq t_{2}$, satisfies the strengthened Clebsch condition if (15.1) holds for almost all $t$ such that $y_{0}^{\prime}(t)$ is defined and is different from $(0, \cdots, 0)$ and for all $v$ orthogonal to $y_{0}^{\prime}(t)$.

(14) E. J. McShane, Curve-space topologies associated with variational problems, Annali della R. Scuola Normale Superiore di Pisa, (2), vol. 9 (1940), pp. 45-60.

UNIVERSITY OF VIRGINIA,

Charlottesville, Va. 